This item was submitted to Loughborough's Research Repository by the author.

Items in Figshare are protected by copyright, with all rights reserved, unless otherwise indicated.

\title{
Computational fluid mixing
}

PLEASE CITE THE PUBLISHED VERSION

PUBLISHER

(C) Wiley Blackwell

VERSION

SMUR (Submitted Manuscript Under Review)

LICENCE

CC BY-NC-ND 4.0

REPOSITORY RECORD

Rielly, Chris D., and Jolius Gimbun. 2009. "Computational Fluid Mixing”. figshare. https://hdl.handle.net/2134/5446. 
This item was submitted to Loughborough's Institutional Repository (https://dspace.lboro.ac.uk/) by the author and is made available under the following Creative Commons Licence conditions.

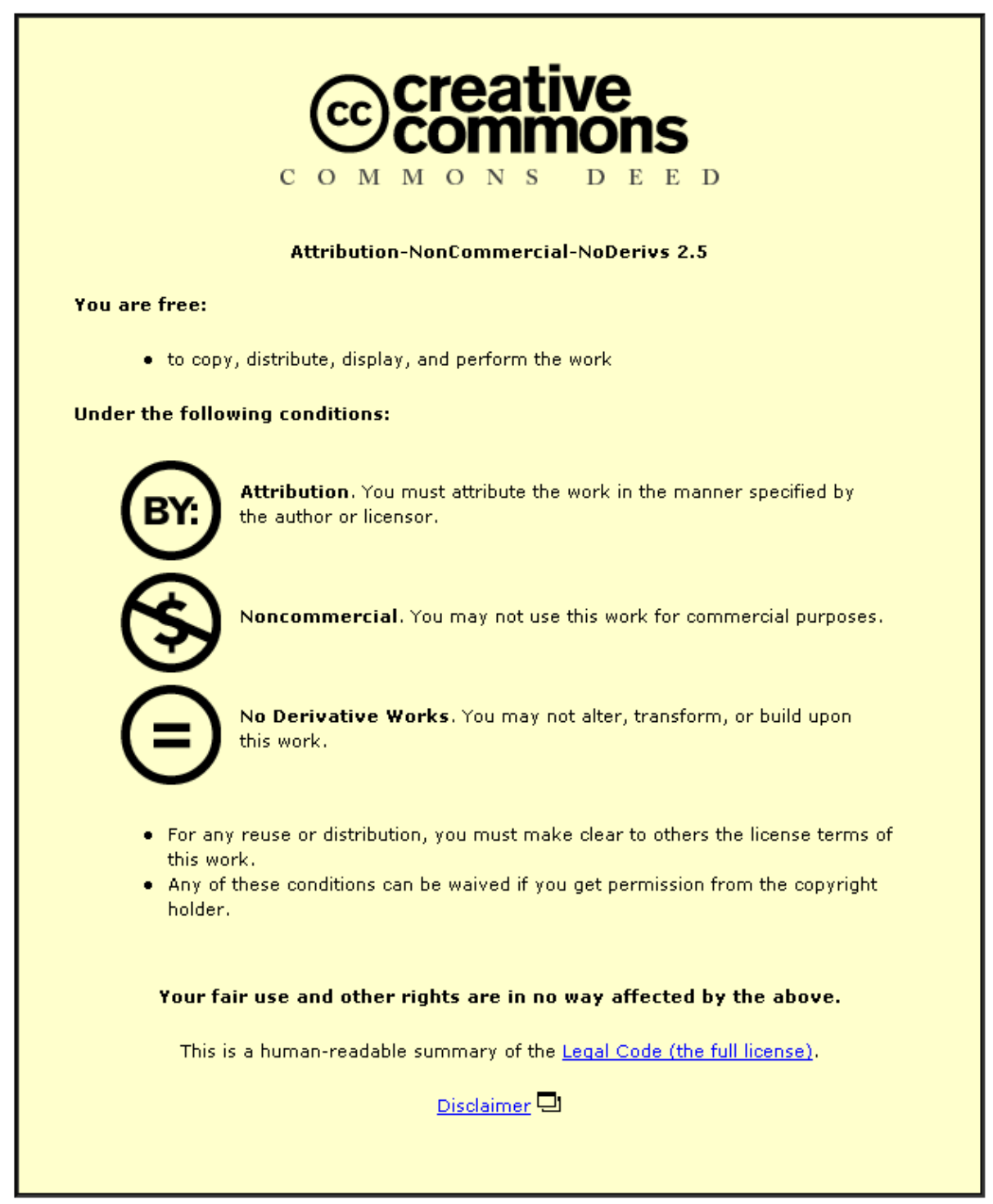

For the full text of this licence, please go to: http://creativecommons.org/licenses/by-nc-nd/2.5/ 


\section{Chapter 8}

\section{Computational fluid mixing}

Chris D. Rielly and Jolius Gimbun

\subsection{Introduction}

\subsubsection{History of CFD}

Computational fluid dynamics (CFD) is an extremely powerful tool for solving problems associated with flow, mixing, heat and mass transfer and chemical reaction. Although the equations of motion for fluid flow were established in the first half of the nineteenth century (e.g. Navier, 1822; Stokes, 1845), it was not until the arrival of digital computers in the 1960s and 1970s that it became feasible to perform numerical simulations of complex engineering flows. In these early days, CFD was a very much a research tool and most of the early work was aimed at developing numerical methods, solution algorithms and Reynolds-averaged turbulence models. However, in the 1980s, the first commercial codes emerged - e.g. PHOENICS, FLUENT, FIDAP, Star-CD, FLOW3D (which later became CFX) — providing general purpose software packages for both academic and industry users. The aerospace and automotive industries were amongst the first to embrace the use of CFD in engineering design, but from the 1990s onwards commercial codes have found widespread applications, for example in: biomedical engineering, environmental and atmospheric modelling, meteorology, chemical reaction engineering and more recently in the food and beverage industries. This chapter will focus on mixing 
vessel applications for the last two of these industry sectors, where CFD is increasingly used to provide process understanding and semi-quantitative analysis.

In their review, Norton and Sun (2006) presented a graph showing the very significant increase in the number of peer-reviewed papers related to CFD applications to food process engineering. Figure 0.1 is an updated version of this graph, containing more recent data and showing that the number of papers that specifically analyse food mixing operations using CFD is still relatively small. In

contrast, there are a vast numbers of papers on CFD simulation of (i) other food process operations, (e.g. drying, sterilisation, thermal treatment and extrusion, many of which are described by Sun (2007)) and (ii) more conventional mixing operations in the chemicals and specialty product industries (see for example, Marshall and Bakker (2004)). This chapter will outline the background knowledge required for CFD studies, present some examples of CFD modelling of mixing vessel flows and finally will discuss the current difficulties in applying this approach to food mixing processes.

\subsubsection{Steps towards CFD simulation of mixing processes}

Although commercial CFD codes provide user-friendly, menu driven front-ends there is substantial work to be performed before any computation is carried, as is shown schematically in Figure 0.2. For a mixing problem, before the software is first fired up, a number of questions should be asked, with the aim of defining suitable physical models and assumptions for the simulation.

- Is the flow, one, two or three dimensional?

- Can simplifications be made to the flow domain, for example, by using planes of symmetry, periodic boundaries, or axisymmetric assumptions?

- Is the flow single phase and / or single component?

- Is the liquid phase Newtonian or non-Newtonian?

- Is the flow laminar, transitional or turbulent?

- Does the flow involve heat and/or mass transfer? 
- Is a detailed model of the impeller required?

- Are unsteady-state dynamics important?

These are important decisions to make, since they will affect the complexity and results of the computations. Judicious choice of simplifying assumptions can lead to drastic reductions in computing time, at the expense of only minor losses of accuracy; alternatively, inappropriate assumptions, or poor selection of models, can produce low quality solutions, with misleading results. The following parts of this chapter will show how these decisions are implemented in a CFD environment. Although commercial CFD codes appear to give the novice user access to powerful tools, they should be used with caution and with expert advice.

The next stage in setting up a simulation is to define material properties and constitutive equations, for example to represent the effects of pressure and temperature on density, or the effects of deformation rate on the apparent viscosity, i.e. the rheological law. It may also necessary to collect data and select equations to represent heat and mass transfer effects and chemical reaction kinetics (simplified kinetic schemes are often required). Information is also required for selection and setting of appropriate boundary conditions for various parts of the flow domain, as is described later in section 8.2.7.

The steps up until this point have mainly involved the collection of data and the selection, based on engineering knowledge, of appropriate constitutive and transport models. The final part of pre-processing is carried out within the software environment and involves

- detailed definition of the internal flow domain, using the dimensions and geometry of the mixer; often this is input or imported through a CAD interface

- generation of a grid, which discretises the flow domain into a large number of control volumes; the grid does not need to have a regular 
structure, can be fitted around complex geometries and can be refined in regions where greater resolution is required (see section 8.3.2)

- input of the fluid properties (often available as part of a library), selection of appropriate models to represent the flow physics and chemistry and input of boundary and operating conditions, based on the information obtained previously and on engineering judgements with regard to suitable assumptions and simplifications

- initialisation of the velocity, pressure and concentration or temperature fields, either as starting guesses for an iterative solution, or as initial conditions for a transient calculation (see section 8.3.4

- $\quad$ selection of suitable numerical schemes, solution monitors and controls; typically software will contain default settings, so that robust, but possibly slow and inaccurate schemes are implemented; improved convergence rates and accuracies may be achieved by expert selection of the numerical schemes, but often this is only done once a partially converged solution has been obtained (see section 8.3.4 and section 8.3.5).

By this point, the problem is fully specified within the computer software, although the user does not see the detailed numerics behind the code, nor is there a requirement for him or her to discretise the differential transport equations which will be solved in the ensuing calculations. Typically the user would then initialise the problem and then set the code running to converge to a solution (or for transient cases, to run for a set period of time). The progress of the solution can be monitored via residuals, which are measures of the errors in solving each of the transport equations (described in section 8.3.5). Most codes can be set to terminate the iterations when the residuals have fallen below some prescribed value; typically default values are supplied by the code vendor, which would mean that the solution was well enough converged, for engineering design purposes.

Convergence of the numerical solution is just one aspect of obtaining an accurate solution-it means that the errors in the conservation balance equations are small, but it does not guarantee that the spatial distribution of velocities, pressures etc. are accurately predicted. The latter should be demonstrated by grid independence studies. The initial grid that was generated is often coarse, but it can 
later be refined (made smaller), so that gradients in the flow are more accurately represented (see for example, the grid independence study reported by Aubin et al., 2004a). If the velocity and pressure fields do not change when the grid is refined, then an accurate, grid-independent solution has been obtained. In cases where the solution does change, then further grid refinement is required. Fluid mechanics know-how should be applied to increase the density of grid points in regions where strong velocity gradients might be expected, e.g. close to walls or moving blades. An example of this type of grid refinement is shown in Figure 0.3: the grid spacing has been reduced around the impeller level, to resolved the strong velocity gradients in this region of the discharge flow, where trailing vortices are attached to each impeller blade. This is a more intelligent use of grid refinement, rather than simply increasing the grid density at all points in the flow and can lead to significant improvements in computationally efficiency. For transient flows, time step independence should be also be demonstrated by decreasing the $\Delta t$ until the solution stops changing. Grid and time step independence studies are laborious calculations, but are necessary to demonstrate the accuracy of the solution.

Commercial CFD codes provide a wide range of post-processing options to display, visualise and analyse the flow solutions. Graphical tools allow visualisation of the flow geometry and grid distribution, as well as output of vector, contour and 3D plots of any of the flow or transport variables. Individual planes or surfaces, or profiles can be plotted and manipulated by rotation, scaling and perspective within the flow domain; transient solutions can be animated for greater visual effect and addition effects, such as particle tracking can be applied to show flow patterns.

Inexpert users often stop at this point and admire their colourful graphical output. However, the job is not yet done. Expert users would attempt to validate 
their CFD model using experimental data, e.g. obtained from small scale equipment and preferably based on local velocity, pressure or temperature measurements.

Chapter 7 discusses some methods for obtaining velocity measurements in small scale flows, namely, laser-Doppler anemometry (LDA) and particle image velocimetry (PIV). Alternatively, some validation could be obtained by comparing global characteristics of the flow with experimental observations, e.g. flow pattern comparison, or flow number, or power number comparisons with literature values (see section 8.4.3). By making such validation studies the user provides an extra check that physical models, simplifying assumptions, numerical schemes and discretisation methods have been appropriately chosen. Successful validation of the CFD model at small scale, generally indicates that accurate solution of the velocity, temperature and pressure fields would be obtained in larger scale, but geometrically similar equipment. Poor agreement with experimental data may suggest ways in which the simulation assumptions or models can be improved.

Finally, as is indicated in Figure 0.2 the reason for conducting the CFD simulation was to answer some questions about the fluid flow, mixing, heat transfer rate, or to design and optimise the performance of a new process. So now the solution must be interpreted and analysed and judgement must be exercised. Hence, it should be obvious all along the way, there is a need for fluid mechanics knowledge and physical insight to define the problem, choose suitable simulation conditions, validate and verify the result and finally interpret the solution to provide an answer to an engineering problem.

So far, a general approach has been described to simulate mixing operations using CFD. The following sections will provide basic information which will be useful in deciding how to implement the various models and methods. Section 8.2 
describes the fundamental transport equations that must be solved for flows with mixing and heat transfer in the laminar and turbulent regimes; selection of suitable boundary conditions is also discussed. The numerical methods to solve these equations are then outlined in section 8.3 and special techniques for handling impeller-driven flows are covered in section 8.4. The latter section also presents a number of examples from the literature of studies which have simulated liquid flows, blending and multi-phase mixing applications. Finally, the challenges for modelling mixing operations related to food processing are discussed and some examples of dough mixing and crystalliser design are reviewed.

\subsection{Conservation equations}

A key hypothesis in dealing with flow and mixing problems is that the fluid behaves as a continuum (Batchelor, 2000), such that local values of the velocity, pressure and density may be defined at a point and these properties change smoothly in space and in time. In other words, the molecular structure of a gas or liquid is not considered explicitly and the fluid is treated as a macroscopic continuum. On this basis, the governing equations of fluid mechanics may be obtained by application of the following fundamental laws:

(i) mass conservation: mass cannot be created or destroyed

(ii) Newton's second law: the rate of change of momentum is equal to the sum of the forces acting on a fluid element

(iii) the first law of thermodynamics: energy can be converted between different forms, but is neither created nor destroyed. 
(iv) chemical species conservation: atomic species are conserved, whereas molecular species can be transformed by chemical reactions, with a prescribed stoichiometry.

Detailed discussion and formulation of these transport equations can be obtained in fluid mechanics text books, such as "Transport phenomena" by Bird, Stewart and Lightfoot (2007). Here the aim is to give the reader an insight into the general structure of these equations, which all essentially take the same form.

Consider the arbitrary control volume, $V$, which is fixed in space and is bounded by a surface $S$, shown in Figure 0.4 . For any scalar property $\Phi$ the general conservation law may be stated as

$\begin{array}{lll}\begin{array}{l}\text { Rate of accumulation } \\ \text { of } \Phi \\ \text { inside } V\end{array} & \begin{array}{l}\text { Net efflux } \\ \text { of } \Phi \\ \text { through } S\end{array} \quad=\begin{array}{l}\text { Sources } \\ \text { of } \Phi \\ \text { inside } V\end{array}-\begin{array}{l}\text { Sinks } \\ \text { of } \Phi \\ \text { inside } V\end{array}\end{array}$

or in terms of mathematics

$$
\int_{V} \frac{\partial(\rho \Phi)}{\partial t} \mathrm{~d} V+\int_{\boldsymbol{S}}\left(\rho \boldsymbol{u} \Phi+\boldsymbol{j}_{\Phi}\right) \cdot \mathrm{d} \boldsymbol{S}=\int_{V} S_{\Phi} \mathrm{d} V
$$

where $\boldsymbol{u}$ is the velocity vector, $\boldsymbol{j}_{\Phi}$ is the diffusive flux of $\Phi$ through surface $S, \rho$ is the fluid density and $S_{\Phi}$ is the sum of sources minus sinks for $\Phi$ inside the control volume $V$. Note that here $\Phi$ is written as an intensive scalar quantity, per unit mass and that transport of $\Phi$ across the boundary can occur by both convection and diffusion. Using Gauss' divergence theorem allows the rewriting of the flux through surface $S$ as the volume integral of a divergence and hence

$$
\frac{\partial(\rho \Phi)}{\partial t}+\nabla \cdot(\rho \boldsymbol{u} \Phi)=-\nabla \cdot \boldsymbol{j}_{\Phi}+S_{\Phi}
$$

where, $\nabla$ is the nabla or del vector operator; for example, in Cartesian coordinates 


$$
\nabla=\left(\frac{\partial}{\partial x}, \frac{\partial}{\partial y}, \frac{\partial}{\partial z}\right)
$$

and $(x, y, z)$ represent the three coordinates. The LHS of eq.(0.3) gives the total rate of change of $\Phi$ in a fluid particle moving at velocity $\boldsymbol{u}$; in fluid mechanics, this is known as the convective derivative or the derivative following the motion:

$$
\frac{\partial(\rho \Phi)}{\partial t}+\nabla \cdot(\rho \boldsymbol{u} \Phi)=\frac{D(\rho \Phi)}{D t}
$$

Almost all fluid mechanics simulations require the application of the general conservation statement of eq.(0.3) to mass and momentum. In addition, if heat transfer occurs, resulting in non-isothermal flows, or chemical reaction takes place, then the conservation equation must also be applied to the energy and chemical species balances.

In applying such equations to predict flow and mixing in chemical and food processing operations, it is often permissible to make some simplifying assumptions. For example, the flows will often involve incompressible liquids, in which case the fluid density may be assumed to be a constant and hence may be moved outside the derivatives of eq.(0.3).

\subsubsection{Mass Conservation}

For mass conservation, there are no sources and sinks and the scalar quantity $\Phi$ is simply equal to unity. Then eq.(0.3) becomes

$$
\frac{\partial \rho}{\partial t}+\nabla \cdot(\rho \boldsymbol{u})=0
$$

which is known as the continuity equation. Flows in mixing vessels generally involve an incompressible liquid ( $\rho=$ constant), in which case eq.(0.6) reduces to 


$$
\nabla \cdot \boldsymbol{u}=0
$$

which applies to both steady and unsteady-state flows. In Cartesian $(x, y, z)$ coordinates, with velocity $\boldsymbol{u}=(u, v, w)^{t}$, eq.(0.7) may be written as

$$
\nabla \cdot \boldsymbol{u}=\frac{\partial u}{\partial z}+\frac{\partial v}{\partial y}+\frac{\partial w}{\partial z}=0
$$

\subsubsection{Momentum Conservation}

Momentum is a vector quantity and so the conservation equation (0.3) must be applied in each of the coordinate directions. For example, the $x$-direction momentum per unit volume is $\rho u$ and hence $\Phi=u$. In this case the LHS of the conservation equation represents the rate of change of $x$-direction momentum of the fluid

$$
\frac{\partial(\rho u)}{\partial t}+\nabla \cdot(\rho \boldsymbol{u} u)=\frac{D(\rho u)}{D t}
$$

and therefore, according to Newton's second law, the RHS of eq.(0.9) must represent the sum of the forces acting on an elemental volume of fluid. The forces acting on a control volume of fluid may be divided into two types: body forces (e.g. gravity) and surface forces (pressure or viscous stress effects). Figure 0.5 and Figure 0.6 show the stresses acting in the $x$-direction, using the notation that $\tau_{i j}$ is the stress in direction $j$ acting on face with a normal in the direction $i$. From these figures it is evident that (i) differences in pressure between the shaded faces give rise to a net force and (ii) differences in viscous stresses on all six faces can result in a contribution to the net force. Hence replacing the RHS of the conservation equation by the forces per unit volume acting on the control volume, yields 


$$
\frac{\partial(\rho u)}{\partial t}+\nabla \cdot(\rho \boldsymbol{u u})=-\frac{\partial p}{\partial x}-\left(\frac{\partial \tau_{x x}}{\partial x}+\frac{\partial \tau_{y x}}{\partial y}+\frac{\partial \tau_{z x}}{\partial z}\right)+\rho g_{x}
$$

or in all three directions, using vector notation

$$
\rho \frac{D \boldsymbol{u}}{D t}=-\nabla p-\nabla \cdot \tau+\rho \boldsymbol{g}
$$

where $\tau$ is the stress tensor. Equation (0.11) is fully rigorous for incompressible fluids, typical of these found in mixing operations. Other than that it is quite general, in that it could be applied to both Newtonian and non-Newtonian fluids, by changing the constitutive equation relating the stress tensor $\tau_{i j}$ to the deformation rate tensor, $S_{i j}$. For example, eq. (0.11) can easily be extended to the special case of an incompressible Newtonian fluid (see Ch. 3), with a constant viscosity coefficient, $\mu$.

$$
\tau_{i j}=\mu S_{i j}=\mu\left(\frac{\partial u_{i}}{\partial x_{j}}+\frac{\partial u_{j}}{\partial x_{i}}\right)
$$

Use of eq.(0.12), along with the incompressible continuity eq.(0.7) leads to the wellknown Navier-Stokes equations

$$
\rho \frac{D \boldsymbol{u}}{D t}=-\nabla p-\mu \nabla^{2} \boldsymbol{u}+\rho \mathbf{g}
$$

Alternatively, other constitutive models may be applied in eq. (0.11) to relate the stress tensor components to velocity gradients in the flow. In these cases, the apparent viscosity, $\mu_{a}$, of the fluid depends on the second invariant of the local deformation rates according to

$$
\mu_{a}=f(\dot{\gamma}) \text { where } \dot{\gamma}=\sqrt{\frac{1}{2} S_{i j} S_{i j}}
$$

where summation is implied over the repeated suffixes $i$ and $j$. In commercial CFD codes, such as FLUENT, a variety of constitutive models are available to describe 
non-Newtonian rheologies in the laminar flow regime, e.g. power law, Carreau model, Cross model and Herschel-Bulkley model for Bingham plastics (shear thinning fluids with a yield stress). Constitutive parameters for these models must be obtained from experimental tests, as described in Chapter 3 on Food Rheology.

\subsubsection{Turbulence}

The conditions for laminar, transitional and turbulent flows may be distinguished according to a dimensional group known as the Reynolds number, which in general may be defined as

$$
R e=\frac{\text { inertial stresses }}{\text { viscous stresses }}=\frac{\rho U L}{\mu}
$$

where $U$ is a characteristic velocity scale and $L$ is a characteristic length scale. In mixer designs, these are conventionally defined in terms of the impeller tip speed (proportional to $N D$, where $N$ is the rotational speed, conventionally in rev/s) and the impeller diameter $D$.

$$
R e=\frac{\rho N D^{2}}{\mu}
$$

Typically in stirred vessels flows the flow is laminar for $R e<10$ and fully turbulent for $R e>10^{4}$. Thus there is a wide range of transitional flow over $10<R e<10^{4}$, where the flow is turbulent around the impeller and relaminarises in the bulk. This is a particularly challenging area for CFD.

Turbulent flows are characterised by three-dimensional, fluctuating velocity components which lead to increased rates of transport for mass, momentum and energy. Although the equations presented in sections 8.2.1 and 8.2.2 are valid for turbulent flow, they must be applied instantaneously and hence required to be solved using very short time and space steps to model the flow accurately. With modern 
computing resources this type of direct numerical simulation (DNS) is possible for some limited cases of low Reynolds number turbulence, but for most practical applications the number of grid and time steps required is orders of magnitude too high to be feasible. Hence a statistical approach, known as Reynolds-averaging is often applied to the description of turbulence quantities (See Versteeg and Malalasekara (2007) for a more detailed account).

The Reynolds-averaged Navier-Stokes (RANS) equations result from decomposing the velocities, $\boldsymbol{u}$, and all other scalar quantities, $\phi$ (pressure, temperature, mass fraction) into mean and fluctuating components.

$$
\boldsymbol{u}=\overline{\boldsymbol{u}}+\boldsymbol{u}^{\prime} \text { and } \Phi=\bar{\Phi}+\Phi^{\prime}
$$

Time-averaging the continuity eq.(0.7) gives

$$
\nabla \cdot \overline{\boldsymbol{u}}=0
$$

whereas, applying the same process to eq.(0.11) generates additional Reynolds stresses, $\overline{\tau_{T}}$ due to the averaging of the non-linear advection terms, $\boldsymbol{u} \cdot \nabla u$

$$
\rho \frac{D \overline{\boldsymbol{u}}}{D t}=-\nabla \bar{p}-\nabla \cdot \overline{\tau_{L}}-\nabla \cdot \overline{\tau_{T}}+\rho \mathbf{g}
$$

As before, the laminar stress tensor is given in terms of the mean velocity gradients by

$$
\left(\overline{\tau_{L}}\right)_{i j}=\mu\left(\frac{\partial \overline{u_{i}}}{\partial x_{j}}+\frac{\partial \overline{u_{j}}}{\partial x_{i}}\right)
$$

However, six new unknown quantities, the Reynolds stresses,

$$
\left(\overline{\tau_{T}}\right)_{i j}=\left(\overline{\tau_{T}}\right)_{j i}=-\rho \overline{u_{i}^{\prime} u_{j}^{\prime}}
$$


have now been introduced, but with no new equations to represent their transport. They represent turbulent momentum fluxes and involve statistical correlations between the various fluctuating velocity components. There is now a turbulence closure problem, with more unknowns than equations and hence the new terms must be approximated by empirical models. One such model is based on the Boussinesq hypothesis which draws an analogy with the laminar stress tensor in eq.(0.20) and proposes

$$
\left(\overline{\tau_{T}}\right)_{i j}=-\rho \overline{u_{i}^{\prime} u_{j}^{\prime}}=\mu_{T}\left(\frac{\partial \overline{u_{i}}}{\partial x_{j}}+\frac{\partial \overline{u_{j}}}{\partial x_{i}}\right)-\frac{2}{3} \rho k \delta_{i j}
$$

where $\mu_{T}$ is a turbulent or eddy viscosity, $k=\frac{1}{2}\left(\overline{u_{i}^{\prime 2}}+\overline{u_{j}^{\prime 2}}+\overline{u_{k}^{\prime 2}}\right)$ is the turbulent kinetic energy per unit mass and $\delta_{i j}=0, i \neq j$ and $\delta_{i j}=1, i=j$ is the Kronecker delta. However, this has not yet solved the turbulence closure problem, as the eddy viscosity is still an unknown and has to be computed from further empirical models. In contrast to the laminar viscosity, $\mu$, the eddy viscosity is not a material property; it depends on strain rates and eddy length scales in the flow and hence is distributed in time and space.

The two-equation $k-\varepsilon$ turbulence model is a popular method to calculate $\mu_{T}$, where $k$ is the turbulence kinetic energy defined above and $\varepsilon$ is its dissipation rate (the rate at which the turbulence kinetic energy is converted to heat by viscous effects at the scale of the smallest eddies — the Kolmogorov scale). The standard $k-\varepsilon$ model calculates the (isotropic) eddy viscosity from

$$
\mu_{T}=\rho C_{\mu} \frac{k^{2}}{\varepsilon}
$$


where $C_{\mu}$ is a dimensionless constant. Approximate transport equations for $k$ and $\varepsilon$ are formulated for incompressible flow as

$$
\begin{aligned}
& \rho \frac{\partial k}{\partial t}+\rho \overline{\boldsymbol{u}} \cdot \nabla k=\nabla \cdot\left[\left(\mu+\frac{\mu_{T}}{\sigma_{k}}\right) \nabla k\right]+2 \mu_{T} S_{i j} S_{i j}-\rho \varepsilon \\
& \rho \frac{\partial \varepsilon}{\partial t}+\rho \overline{\boldsymbol{u}} \cdot \nabla \varepsilon=\nabla \cdot\left[\left(\mu+\frac{\mu_{T}}{\sigma_{\varepsilon}}\right) \nabla \varepsilon\right]+C_{1 \varepsilon} \frac{\varepsilon}{k} 2 \mu_{T} S_{i j} S_{i j}-C_{2 \varepsilon} \rho \frac{\varepsilon^{2}}{k}
\end{aligned}
$$

Whilst the $k$ equation is almost exact, the $\varepsilon$ equation contains a number of modelled terms and empirical constants. The standard $k-\varepsilon$ model contains five such constants

$$
C_{\mu}=0.09, \sigma_{k}=1.00, \sigma_{\varepsilon}=1.30, C_{1 \varepsilon}=1.44, C_{2 \varepsilon}=1.92
$$

which have been tuned by data fitting experiments conducted in turbulent shear flows and grid generated turbulence. It may be tempting to alter these empirical constants to obtain better agreement with experimental results, but this not recommended as a good practice (Versteeg and Malalasekara, 2007).

Thus, with such a model, the transport equations $(0.24)$ and $(0.25)$ may be solved to give the distributions of $k, \varepsilon$ and the eddy viscosity via eq.(0.23). Hence the unknown Reynolds stresses may be calculated from eq.(0.22) for inclusion in the RANS eqs.(0.19).

Many variants of the standard $k-\varepsilon$ model have been proposed, amongst which are:

- the RNG $k-\varepsilon$ model was obtained from renormalization group theory by Yakhot, and Orszag, (1986) and has the following features: (i) a strain dependent term was added in the $\varepsilon$ equation which improves its accuracy for rapidly strained flows, such as bends and expansions; (ii) the effects of swirl are more accurately 
modelled, which are important feature of many mixing flows; (iii) the effective viscosity is obtained from a differential equation, which better accounts for low Reynolds number effects and hence is better suited to transitional flows.

- the realizable $\boldsymbol{k}-\varepsilon$ model was proposed by Shih (1995) and differs from the standard form in that it has (i) a new expression for the calculation of the eddy viscosity, with a variable value of $C_{\mu}$ and (ii) entirely different source and sink terms for the $\varepsilon$ equation. It is claimed to be superior for flows that involve the spreading of planar and round jets (e.g. as in the discharge stream from an impeller) and for flows with rotation and recirculation.

In contrast, other RANS turbulence models do not make use of the Boussinesq hypothesis, with its in-built assumption of an isotropic eddy viscosity. Instead the Reynolds Stress model (RSM) solves individual transport equations for each of the six independent Reynolds stresses, plus a further conservation equation for the dissipation rate (in contrast, the standard $k-\varepsilon$ model only solves an extra two equations to simulate turbulent flows). The model is too complex to present in detail here, but is discussed further by Versteeg and Malalasekara (2007). As before, the closure problem forces some of the terms in the RSM equations to be modelled by empirical means. The greater number of transport equations to be solved in the RSM significantly increase the computational overhead of performing the calculations. In principle, RSM models should give improved predictions for flows that involve swirl, significant streamline curvature and rapid straining, i.e. where an isotropic eddy viscosity assumption is invalid. In practice, as is described in section 8.4.5, they have not offered great improvement for stirred mixer simulations.

Although a large number of RANS based approaches have been proposed, there is no one general model that is capable of producing accurate turbulence 
descriptions for a wide variety of flows. This is partly down to the difficulty in modelling both large eddies, whose direction is dependent on the geometry of the flow, and small eddies, who tend to have a more universal and isotropic character (see for example Kolmogorov's (1941) local isotropy assumption, which is valid for high Reynolds numbers, for a developed inertial sub-range of the energy spectrum). A more recent approach is to resolve numerically the largest eddies in a timedependent calculation and only to model the smallest eddies, using for example the Boussinesq hypothesis. This is the basis of Large Eddy Simulations (LES), which use spatial filtering and a cut-off width to separate large and small eddy effects. The resulting filtered Navier-Stokes equations may then be used to calculate explicitly the unsteady velocities of the large scale eddies (larger than the cut-off width), whereas the small scale eddies are represented through a sub-grid scale stress model, based on the eddy viscosity concept.

Figure 0.7 represents schematically the hierarchy of approaches to turbulence modelling. Direct numerical simulation uses fantastically detailed numerical simulation to resolve in time and space the motions of even the very smallest, Kolmogorov eddies in the flow, but is generally unsuitable for practical application. At the other extreme, RANS models predict only time-averaged quantities of the flow; whilst they can be tuned for certain flow pathologies, they are essentially empirical in nature and liable to provide less accurate results for flows in mixing vessels, which involve strong streamline curvature, flow separation and rapid straining. The compromise is to use LES, with a filtering scale chosen to represent the important eddy sizes; even still, practical applications at high Reynolds numbers will still involve lengthy computations, especially for wall-bounded flows. 
LES is an excellent approach away from the boundary layer, however it may not yield sufficient resolution of the near wall flow structure, especially at high Reynolds numbers because the large eddies close to the wall are physically small in size. It is possible to resolve this near wall flow structure using a very fine grid but this will make the mesh requirement (and hence the computational demand) almost equivalent to that of DNS (Spalart et al., 1997). Inadequate grid resolution of boundary layers can severely degrade the LES approximation and thus separated flows may not be predicted accurately. To solve this problem, Spalart et al. (1997) proposed a new turbulence model called detached eddy simulation (DES) which combine both the RANS and LES model. The main idea is to combine RANS modelling with LES for applications in which the classical LES is not affordable i.e. in the boundary layers. DES reduces to RANS model in boundary layer, thus permitting a coarser grid than for conventional LES, resulting in fewer overall mesh points and faster computation. This approach retains the full sensitivity of RANS model predictions around the boundary layer and the LES away from the wall. Despite its potential the DES so far has not yet been used for simulating the mixing tanks.

\subsubsection{Energy Conservation}

Non-isothermal flows may be modelled by solving an additional equation, based on conservation of energy. This would be necessary for flows that involve (i) heat transfer from jackets or coils; (ii) chemical reaction which releases or consumes heat and (iii) considerable energy dissipation, which converts mechanical energy to heat through viscous effects. High viscosity fluids, such as bread dough, are likely to experience significant temperature rises during mixing because of viscous dissipation, whereas for low viscosity solutions, the flows can often be treated as isothermal. 
For energy conservation, the quantity $\Phi$ in eq.(0.3) is equivalent to the specific energy which is conveniently written as $C_{p} T$. The conductive heat flux is usually represented by Fourier's law which leads to

$$
\rho c_{p} \frac{\partial T}{\partial t}+\rho c_{p}(\boldsymbol{u} \cdot \nabla T)=\kappa \nabla^{2} T+Q+E
$$

where $\kappa$ is the thermal conductivity, $Q$ is a volumetric rate of heat generation (e.g. from electrical resistance heating, or from chemical reaction) and the term $E=-\tau: \nabla u$ represents viscous dissipation of heat. In writing eq.(0.27), it has been assumed that the density, specific heat capacity and thermal conductivity of the fluid are constants and independent of temperature. Generally, commercial software codes will use a slightly more complex form of the energy equation which allows for temperature dependent physical and transport properties.

In tackling non-isothermal flow problems it is worth considering how strongly coupled the temperature field is with the flow field. In many cases where forced convection dominates, the dissipation term is negligible compared to the enthalpy transport terms and hence the energy equation is effectively uncoupled from the momentum equations. In such a case, the velocity and pressure fields can be converged to a solution first, without considering temperature variations. Then the energy equation can be switched on and solved simultaneously independently of the momentum and continuity equations. On the other hand, for flows involving natural convection, the effects of temperature dependent properties are important, and there is strong coupling between the energy and momentum equations. These types of considerations determine the order in which the equations are solved in CFD and the requirements to solve them independently or simultaneously. 


\subsubsection{Species Transport}

In chemically reacting flows, mixing operations, or flows with mass transfer, the mass fractions, $Y_{i}$ of the various components within a mixture may be calculated from a differential mass balance

$$
\rho \frac{\partial Y_{i}}{\partial t}+\rho\left(\boldsymbol{u} \cdot \nabla Y_{i}\right)=D_{i m} \rho \nabla^{2} Y_{i}+R_{i}+S_{i}
$$

where $D_{i m}$ is the molecular diffusivity of species $i$ in the liquid solvent, $R_{i}$ is a rate of production of $i$ per unit volume by chemical reaction and $S_{i}$ represents other volumetric source terms, e.g. though inter-phase mass transfer. For a mixture containing $C$ components, there are $C-1$ independent species transport equations, since the mole fractions must add to unity. The FLUENT manual (Fluent, 2005) suggest that accuracy is best obtained by omitting the species with the largest mass fraction (often the solvent or carrier liquid phase). Equation (0.28) is based on assumption that diffusion may be modelled using Fick's law, which will be suitable for either binary cases, or where the solutes are at low concentration in the liquid phase.

\subsubsection{Turbulent species and energy transport}

In turbulent flows, Reynolds-averaging of the transport equations (0.27)and (0.28) leads to additional fluxes in the energy and species conservation equations, due to averaging of the non-linear advection terms, such as $\boldsymbol{u} \cdot \nabla T$. The extra fluxes are precisely why mixers are designed to operate in turbulent flow. As before the velocities and/or temperatures and mass fractions are written in terms of mean and fluctuating quantities, e.g. in addition to eq.(0.17)

$$
T=\bar{T}+T^{\prime} \text { and } Y_{i}=\bar{Y}_{i}+Y_{i}^{\prime}
$$


Then, following application of the Boussinesq hypothesis, eqs.(0.27) and (0.28) become

$$
\begin{aligned}
& \rho c_{p} \frac{\partial \bar{T}}{\partial t}+\rho c_{p}(\overline{\boldsymbol{u}} \cdot \nabla \bar{T})=\left(\kappa+\frac{\mu_{T} c_{p}}{\operatorname{Pr}_{T}}\right) \nabla^{2} \bar{T}+\bar{Q}+\bar{E} \\
& \rho \frac{\partial \bar{Y}_{i}}{\partial t}+\rho\left(\overline{\boldsymbol{u}} \cdot \nabla \overline{Y_{i}}\right)=\left(D_{i m} \rho+\frac{\mu_{T}}{S c_{T}}\right) \nabla^{2} \bar{Y}_{i}+\bar{R}_{i}+\bar{S}_{i}
\end{aligned}
$$

where the default value of the turbulent Schmidt number is $S c_{T}=0.7$ and for the turbulent Prandtl number is $\operatorname{Pr}_{T}=0.85$. Seeing these conservation equations together, it becomes obvious that for turbulent flow the eddy transport mechanism is the same for momentum, heat and mass; the eddy thermal and mass diffusivities are approximately equal to the eddy kinematic viscosity. Thus, the eddy diffusivities for mass and heat are given by, respectively,

$$
D_{i T}=\frac{\mu_{T}}{\rho S c_{T}} \text { and } \alpha_{T}=\left(\frac{\kappa}{\rho c_{p}}\right)_{T}=\frac{\mu_{T}}{\rho P r_{T}}
$$

\subsubsection{Boundary conditions}

The transport partial differential equations presented in the previous sections represent the general governing equations for incompressible liquid flows with heat and mass transfer. It is the boundary conditions that are imposed on each equation that makes them specific to different flow geometries and operating conditions. This section considers a range of boundary conditions that may be required to simulate flows in mixing vessels.

The vast majority of boundaries in mixing vessel flows are impermeable walls, at which the no slip condition may be applied: 


$$
\boldsymbol{u}=\boldsymbol{u}_{w}
$$

where $\boldsymbol{u}_{w}$ is the wall velocity vector (tank walls and baffles would have zero velocity, but the walls that constitute the surfaces of the impeller blades and shaft have a nonzero velocity). For the energy and species balance equations, wall boundary conditions can be of two types (show here for temperature): (i) Dirichlet boundary condition

$$
T=T_{w}
$$

where $T_{w}$ is a specified temperature at each point on the wall, and (ii) Neumann boundary condition

$$
\left.\kappa \frac{\partial T}{\partial n}\right|_{w}=-q_{w}
$$

where the direction $n$ is normal to the wall, and $q_{w}$ is a specified heat flux, For example, for insulated walls, $q_{w}=0$, or for heat transfer to an external environment at temperature $T_{e x}$, then $q_{w}=h_{e x}\left(T_{w}-T_{e x}\right)$.

For fully baffled stirred vessels, the free surface is reasonably flat and hence is often modelled using a symmetry boundary condition, in which the normal velocity component and the tangential velocity gradients are set to zero.

$$
u_{n}=0 \text { and } \frac{\partial u_{t}}{\partial n}=0
$$

The latter means that the shear stress on the symmetry surface is zero, which is why it corresponds approximately to a free surface condition (effects such as surface tension are not included).

In stirred tank simulations it is also possible to reduce computation times for steady-state cases, by use of cyclic boundary conditions, making use, for example, 
of the four-fold symmetry of a fully baffled vessel. For steady cases (e.g. in RANS simulations, see section 8.2.3 above), the flow exhibits 4-fold symmetry and therefore only one quadrant of the mixing tank needs to be considered. Figure 0.8 shows one quadrant of a stirred vessel, containing one of the four wall baffles. The two planar faces of the quadrant are set as cyclic boundary conditions: the velocity at each $(r, z)$ position leaving the left hand plane is set equal to that arriving at the right hand plane, at the equivalent position.

$$
\boldsymbol{u}(r, z, \theta=0)=\boldsymbol{u}\left(r, z, \theta=90^{\circ}\right)
$$

The preceding discussion covers the range of boundary conditions that would be required for batch mixing operations. For continuous flow operations, boundary conditions are also required for liquid inlets and outlets. Inlet boundary conditions are simply specified in terms of the inlet velocity distribution, temperature and mass fraction of the various species $i$

Inlet b.c. $\quad \boldsymbol{u}=\boldsymbol{u}_{\text {in }}, T=T_{\text {in }}$, and $Y_{i}=\left(Y_{i}\right)_{\text {in }}$

The inlet conditions may be specified using uniform values of $\boldsymbol{u}_{i n}, T_{\text {in }}$ and $\left(Y_{i}\right)_{\text {in }}$ or using inlet profiles, if they are known. Generally the pressure does not need to be set at an inlet condition, when the inlet velocity is specified. Occasionally, the inlet velocity will not be known, in which case it will be necessary to specify the inlet pressure and a pressure at the outlet; in this case, the mass flow rate of liquid entering the tank will adjust to match the imposed pressure difference. For turbulent flow calculations using RANS models, inlet boundary conditions for $k$ and $\varepsilon$ are also required. An inlet value of $k$ can be estimated from typical turbulence intensities, $I$, of around $5 \%$ for pipe and channel flows and $\varepsilon$ can be estimated using a length scale which is proportional to the diameter of the inlet pipe, $D_{i n}$. 


$$
k=\frac{3}{2}\left(\left|u_{i n}\right| I\right)^{2} \text { and } \varepsilon_{\text {in }}=C_{\mu}^{3 / 4} \frac{k_{\text {in }}^{3 / 2}}{0.07 D_{\text {in }}}
$$

where $C_{\mu}=0.09$ is the empirical constant used in eq.(0.23).

Care needs to be taken in locating outlet boundaries, since typically for incompressible flow the boundary condition that will be imposed is zero exit gradient for the velocities, temperature or mass fraction

Outlet b.c. $\quad \frac{\partial u}{\partial n}=0, \frac{\partial T}{\partial n}=0$ and $\frac{\partial Y_{i}}{\partial n}=0, T=T_{\text {in }}$, and $Y_{i}=\left(Y_{i}\right)_{\text {in }}$ where $n$ is the direction normal to the outlet boundary. Placing outlet boundaries where the velocity gradients are unlikely to be zero (or where recirculation advects materials back into the flow domain) is likely to generate erroneous results.

\subsection{Numerical Methods}

\subsubsection{Discretised solution of the flow variables}

The partial differential equations which represent the various conservation statements, described in section 8.2 , can only be solved analytically for a very restricted set of cases, e.g. for some classes of one-dimensional laminar flow. Analytical solutions produce exact functional forms for the dependence of velocity and pressure on time and spatial position. Hence they have no limits of resolution, since these functions can be evaluated at any time or space position. In practice, however, for multi-dimensional and / or unsteady flows, the equations of motion must be solved by numerical means. These methods involve the discretisation of the transport equations, so that the partial derivatives are replaced by algebraic expressions written in terms of discrete values of, e.g. the velocity and pressure, at a 
finite number of positions in space and time. These spatial locations are distributed on the nodes of a grid which is generated by the software to fill the flow domain. The values of the flow variables $\left(p, \boldsymbol{u}, T\right.$ and $\left.Y_{i}\right)$ at the interior grid points of the flow domain are treated as unknowns, whereas those on the boundaries are either known, or can be related to interior points (depending on whether Dirichlet or Neumann boundary conditions have been implemented; see section 8.2.7). Thus the set of partial differential equations that define the physics and chemistry of the flow system, are approximated by a much larger set of algebraic equations (one for each grid position), which must be solved simultaneously to yield the discrete values of the flow variables at the grid points. Similarly, for unsteady-state problems, integration through time is advanced by making a series of finite time steps, i.e. the flow variables are evaluated at discrete times, as well as discrete spatial positions.

\subsubsection{Grid generation}

Commercial CFD software packages contain a front-end which allows the user to specify the flow geometry, typically through a CAD type interface. The flow domain is then divided into (i) sub-volumes, in which a grid is generated, and (ii) surfaces, on which boundary conditions will be applied. Grid generation is carried out by the software, under user control, and is one of the most important and time-consuming parts of conducting a CFD simulation. Construction of a high quality grid will make for a more efficient and robust numerical solution. A high quality grid will have

- $\quad$ sufficient density of cells to capture the important flow gradients

- $\quad$ cells which change slowly and smoothly in size (volume and length)

- $\quad$ cells which are not skewed and have an aspect ratio of around unity 
Early CFD simulations used structured grids, based on simple coordinate systems and had limited ability to mesh around complex geometries. More recently unstructured grids and multi-block grids have allowed a mesh to be generated inside most geometries.

Structured grids are generated by dividing a single, or multiple blocks, of the flow volume into hexahedral elements, which can be logically addressed using $(i, j, k)$ indices in the computational domain (these indices do not need to align with the coordinate system, but the solution will be more accurate if they are aligned with the flow direction). This structure reduces the memory size required, allows for more efficient numerical solution and permits higher-order discretisation scheme (i.e. QUICK, third order) hence a greater accuracy which is not applicable to the unstructured grid. Moreover, the hexahedral elements can tolerate greater skewness and elongation without affecting the stability of the solution and they are subject to smaller numerical diffusion errors than tetrahedral elements. They are however not suited to all geometries and can require considerable user skill to generate satisfactory distributions of the grid points

Unstructured grids are based on dividing the volume into hexahedral or tetrahedral elements which are fitted to the geometry of the flow domain. They are easier to generate automatically for arbitrary geometries: typically the user defines the mesh spacing on the boundaries and the software generates the distribution of internal grid points. Unstructured grids require more memory and have longer computation times than structured grids. Figure 0.9 shows a simple example of meshes generated inside a cylindrical flow domain using either hexahedral or tetrahedral cells. Hybrid grids combine the best features of both structured and unstructured grids, making use of both hexahedral and tetrahedral elements in different blocks within the 
flow domain. The grids points do not need to match each other at the interfaces between blocks, which allows greater flexibility in matching the grid and the flow geometry.

\subsubsection{Discretisation}

Converting the partial differential equations of section 8.2 to a set of algebraic equations is achieved by a process known as discretisation. There are three basic ways to discretise the transport equations: finite difference, finite volume and finite element. Although the latter exhibit more stability than finite volume methods (Huebner et al, 1995), they have mainly found applications in specialist codes, such as those for non-Newtonian and visco-elastic flows, e.g. POLYFLOW. Finite differences are amongst the oldest methods of approximating partial differential equations, but they suffer from the problem that (i) conservation of scalar quantities is not necessarily achieved, unless great care is taken and (ii) they are restricted to simple cell element geometries. The finite volume method overcomes both of these disadvantages, which is why it is widely applied in commercial CFD packages (e.g. PHOENICS, FLUENT, CFX). Within the software the expert user is able to choose details of the numerical scheme to be applied. Formal discretisation of the transport equations by the user is not required, since the software does this automatically for either the default, or user-specified, schemes.

\subsubsection{Finite-volume discretisation methods}

As an example of discretisation based on the finite volume method, consider a simplified steady-state 1-D transport equation for a conserved scalar quantity $\Phi$, with a diffusion coefficient $\Gamma_{\Phi}$ and a volumetric source term $S_{\Phi}$ 


$$
\frac{\partial}{\partial x}(\rho u \Phi)=\frac{\partial}{\partial x}\left(\Gamma_{\Phi} \frac{\partial \Phi}{\partial x}\right)+S_{\Phi}
$$

The control volume in Figure 0.10 is centred at node $P$, at which all the flow variables are stored. Here the quantity $\Phi$ is advected into, or out of, the control volume from neighbouring nodes $W$ and $E$, through the faces labelled $w$ and $e$. The finite volume approximation ensure that $\Phi$ remains properly conserved by first integrating the transport equation over the control volume, making use of the divergence theorem to convert volume integrals to fluxes through surfaces.

$$
\left[(\rho u \Phi)_{e}-(\rho u \Phi)_{W}\right] A=\left[\left(\Gamma_{\Phi} \frac{\partial \Phi}{\partial x}\right)_{e}-\left(\Gamma_{\Phi} \frac{\partial \Phi}{\partial x}\right)_{W}\right] A+\int_{V} S_{\Phi} d V
$$

The terms on the left-hand side represent the net advective fluxes of $\Phi$ leaving through faces $e$ and $w$, whereas the first two terms of the right-hand side represent the diffusive fluxes. These fluxes at the faces $e$ and $w$ have to be determined in terms of the flow variable values stored at the nodes $E$ and $W$; the source volume integral in eq.(0.42) can be written simply as $S_{P \Phi} V_{P}$, where $S_{P \Phi}$ is a linearised function of $\Phi_{P}$ and $V_{P}$ is the volume of cell $P$. The gradients in the diffusive flux terms can be represented by central differences to give second-order accurate schemes.

$$
\left(\frac{\partial \Phi}{\partial x}\right)_{e}=\frac{\Phi_{E}-\Phi_{P}}{x_{E}-x_{P}}
$$

The advective fluxes are related to $\Phi$ values at the nodes by various interpolation schemes, depending on the local Peclet number, $P e=\rho u L / \Gamma$, using

- $\quad$ central differencing $(P e=0)$ for pure diffusion

- $\quad$ upwind differencing $(P e>>1)$ for advection dominated flows

- $\quad$ hybrid and power-law differencing $(0<P e<10)$ 
- $\quad$ higher-order methods, e.g. QUICK (Leonard, 1979) based on weighted averages of second-order upwind and central interpolations, is more accurate on structured grids.

First-order differencing using upwind or power-law schemes are often suitable for flows that are aligned with the grid direction, but for the complex 3D flows in mixing vessels, this is unlikely to happen. Therefore, second-order schemes should be employed to yield more accurate results (although at greater computational expense and reduced stability) for a given grid spacing. To overcome some of the stability issues, it is good practice initially to start CFD mixing simulations using a first-order scheme and run a few iterations to achieve a partially converged solution. Then the differencing is switched to a second-order scheme and the simulation is run until fully converged.

\subsubsection{Solver methods}

Whatever differencing scheme is chosen, the result is that eq.(0.42) can be linearised and written in terms of the $\Phi$ values at neighbouring points (the number depends on the form of grid chosen). In the simple example of Figure 0.10, the resulting equation would be

$$
a_{P} \Phi_{P}=a_{E} \Phi_{E}+a_{W} \Phi_{W}+S_{\Phi c}
$$

where $a_{P}, a_{E}$ and $a_{W}$ are coefficients that can be written in terms of known quantities and $S_{\Phi c}$ is a constant related to the source terms. Even for a multidimensional flow problem, an equation similar to eq. $(0.44)$ can be written, expressing $a_{P} \Phi_{P}$ as a linearised sum of values at its neighbouring nodes.

$$
a_{P} \Phi_{P}=\sum_{\text {neighbours }} a_{i} \Phi_{i}+S_{\Phi c}
$$


There will be one such algebraic equation for each grid node and for each conserved quantity in the flow. This large, but sparse, system of linear algebraic equations, must be solved simultaneously to give the discrete values of each flow variable on all the grid points. For small mesh sizes, a solution by matrix inversion may be feasible, but for realistic CFD simulations containing several thousand grid points, an iterative solution is required, e.g. using the Gauss-Siedel method (Versteeg and Malalasekara, 2007). The iterative solution proceeds slowly, but is susceptible to divergence and hence the changes in the calculated values of $\Phi$ have to be controlled, using under-relaxation. After each iteration, the old value of scalar $\Phi^{\text {old }}$ is updated by adding the required change $\Delta \Phi$ multiplied by an under-relaxation factor $\alpha_{\Phi}$ :

$$
\Phi^{\text {new }}=\Phi^{\text {old }}+\alpha_{\Phi} \Delta \Phi
$$

For $\alpha_{\Phi}=1$, the value of $\Delta \Phi$ would give a new $\Phi^{\text {new }}$ that exactly satisfied eq.(0.45) at that grid point, but this can lead to too rapid changes and divergence. Use of under-relaxation with $0.1<\alpha_{\Phi}<1$ leads to a slower, but more stable convergence. Usually, small under-relaxation factors would be set for each variable during the initial stages of iteration to avoid divergence problems. Then as a converged solution is approached the under-relaxation factors can be increased for faster convergence. At each iteration, the residual or the error in eq.(0.45) can be computed and summed over all cells to monitor the progress towards convergence

$$
R=\sum_{\text {cells }} R_{P} \text { where } R_{P}=\left|\sum_{\text {neighbours }} a_{i} \Phi_{i}+S_{\Phi c}-a_{P} \Phi_{P}\right|
$$

The magnitude of $R$ depends on the scalar variable $\Phi$ being examined and therefore it is usual to normalise the residuals in some way, e.g. by dividing by the residual 
after a small number of iterations (typically 5). For velocity components and pressure, an engineering quality solution would obtained when the normalised residuals fall below $10^{-3}-10^{-4}$.

Commercial packages offer a choice between two basic types of iterative solution:

- $\quad$ segregated solver: each flow variable $(u, v, w$ and $p)$ is solved sequentially over the whole grid for each iterative step

- $\quad$ coupled solver: at each iteration, the flow variables $(u, v, w$ and $p)$ are solved together for each cell, before moving to other cells in the grid

The coupled solver requires more memory than the segregated solver, but the number of iteration needed for convergence can be a lot smaller. In terms of solution accuracy the difference is not substantial for a well converged solution. The segregated solver approach is appropriate for incompressible flows, such as those found in fluid mixing applications. For fluid flow problems, there are four unknowns ( 3 velocity components and pressure: $u, v, w$ and $p$ ) and four equations (3 momentum equations and continuity). The segregated solver starts from guessed values of the velocity and pressure fields (or using values from the previous iteration) and sequentially computes the new values of the velocity components from the momentum equations. However, these are not likely to satisfy the remaining continuity equation and there is a further problem because there is no natural equation that can be used to update the pressure. A standard way around this difficulty is to use the SIMPLE (Semi-Implicit Method for PressureLinked Equations) algorithm by Patankar (1980), which calculates a correction term based on the continuity equation to compute the updated pressure value. This 
ensures both convergence of the pressure field and that mass conservation is satisfied. Additionally, commercial codes will offer alternative pressure-velocity coupling methods, such as SIMPLEC (SIMPLE-Consistent; Vandoormaal and Raithby, 1984) which may improve convergence for some turbulent flow problems and PISO (Pressure-Implicit with Splitting of Operators; Issa, 1986) for transient flow simulations.

The final stage before performing an iterative solution is to provide the starting guesses for all the flow variables. This is a process known as initialisation and involves specifying values of, say, $u, v, w$ and $p$ at all grid locations, or in each subvolume, in the flow domain. Often these starting guesses would all be zero; alternatively known approximate values can be patched in at appropriate grid positions to reduce the number of required iterations or to force the flow in a particular direction. Once initialisation is complete iteration can proceed until convergence has been achieved. It is also more convenient to prepare separate coarse and fine grids for a given geometry. The initial simulation may be carried out using the coarser grid and its result are then used to initialise the fine grid. Such a practice is the first step in checking grid dependence and can minimise the overall computation time.

\subsection{Application of CFD to stirred tank modelling}

\subsubsection{Mixing operations}

Stirred tank mixers typically comprise cylindrical vessels, with dished bottoms, containing a centrally located shaft and one or more impellers; in turbulent flows, the 
tank should be equipped with four wall baffles, which are designed to convert the swirling flow generated by the impeller to axial and radial flow components, thereby giving improved top-to-bottom mixing. In cases, where the aspect ratio (height to diameter ratio) is greater than 1 , the mixing can be improved by using two or more impellers operating on the same shaft; these impellers do not need to be of the same diameter or design and could fulfil quite different agitation duties. For low Reynolds number $(R e<10)$, laminar flows, the motion generated by the impeller is highly damped by viscous effects and does not extend far from the blades. In these cases, large diameter impellers are required that pass close to the walls of the tank. Hence baffles are unnecessary and in fact make mixing worse: regions of almost stagnant flow are found in front and behind the wall baffles and they limit the volume of fluid that can be visited by a large diameter impeller.

Prior to the application of CFD, mixing operations were generally designed making use of empirical rules based on data gained from small scale experimental investigations. The most successful rules have been formulated using dimensional analysis, to scale-up results obtained on geometrically similar vessels and impeller systems. Often, however, there are uncertainties in deciding a suitable scale-up rule and hence errors can occur in extrapolating the results to large, industrial scale vessels. Moreover, with this approach it is not possible, to predict or optimise the tank and impeller design using different geometries and it would be expensive and time-consuming to investigate all these possibilities through experimental studies; Therefore the potential of CFD is to allow the engineer to explore a much wider design space, by simulating a variety of geometries and operating conditions and predicting the process outcome. There still will be a need for experimental validation and verification, but once this is completed, there should be confidence that 
simulations conducted for similar cases, or different scales of operation, should be accurate.

\subsubsection{Representation of the impeller}

Modelling of turbulence flows in stirred tanks is difficult because the flow structures are often turbulent, highly three-dimensional and cover a wide range of spatial and temporal scales. These flows are transient, since trailing vortices are formed in the wake of the blades, so that the flow varies periodically close to the impeller (Yianneskis et al., 1987). In addition, stirred tanks containing wall baffles or fixed internals, such as heating coils or dips pipes are challenging to simulate using CFD, because the shape of the flow domain is not fixed in time. As the impeller rotates relative to the stationary walls and baffles, the geometry of the flow changes; in a fixed inertial frame of reference the flow is unsteady, due to the periodic passage of the impeller blades. Special precautions must be used to generate a grid, which discretises the changing fluid volume and which captures the impeller motion. Adaptive grids, which change with the moving boundaries of the flow domain, are feasible to construct (see section 8.5.2), but there are better and more efficient approaches used in the field of stirred tank modelling.

Before the mid 1990s most stirred tank CFD models made use of a black-box approach to represent the impeller. An internal boundary was defined to coincide with the swept surface of the impeller and boundary conditions for the mean velocity components and turbulence quantities ( $k$ and $\varepsilon$ ) were imposed at specified inlets to represent the discharge flow; the suction sides of the swept surface were set as outlet boundary conditions, as indicated in Figure 0.11 . Although this is a very simple approach, it requires detailed experimental data for all the flow variables in close proximity to the blades. Mean velocities and $k$ values may be obtained, from 
laser-Doppler anemometry measurements (e.g. Derksen et al., 1999), or in more recent times from particle image velocimetry (e.g. Khan et al., 2006); $\varepsilon$ values are much more difficult to measure and may have to be estimated indirectly from the turbulence kinetic energy and an assumed length scale, e.g. Wu and Patterson (1989)

$$
\varepsilon=A \frac{k^{3 / 2}}{\left(3 \Lambda^{2}\right)^{1 / 2}}
$$

where $A=0.85$ is a constant and $\Lambda$ is the integral length scale, estimated as $W / 2$ and $W$ is the impeller blade width.

The $(\bar{u}, \bar{v}, \bar{w}, k, \varepsilon)$ experimental data are tangentially-averaged and timeaveraged and applied as inlet conditions to the boundary. As shown in Figure 0.11, for the case of a Rushton disk turbine, they represent the discharge stream as a axisymmetric swirling radial jet; for other impeller types, different surfaces of the swept volume would be defined as inlet and outlet flows, to represent the discharge and suction sides, respectively. However, since the data are time-averaged there is no possibility to include any details of the trailing vortex structure which follows each blade - such structures were first observed experimentally by van't Riet \& Smith (1975) and characterised by Yianneskis and Whitelaw (1993); they have an important effect on power reduction and the breakage of drops and bubbles.

Although the method has short computation times and yields fairly accurate predictions of the mean flow patterns (e.g. Ranade \& Joshi, 1990; Kresta \& Wood, 1991), it is time consuming to collect experimental data for new impellers and for each change in geometry or tank dimensions. Moreover these data cannot be obtained for large scale vessels, or for multi-phase flows, which rather restricts the application of the method to small scale, single phase operations. Therefore the 
black-box approach is not an a priori design tool and has now been superseded by techniques based on moving meshes.

A better and more explicit approach is to generate an accurate 3D model of the impeller geometry and to mesh this with a grid that moves at the impeller rotational speed (Marshall and Bakker, 2004). For an unbaffled vessel (with no tank internals), the flow is steady-state in a rotating frame of reference with a rotational speed that matches the impeller $N(\mathrm{rev} / \mathrm{s})$. Thus in the rotating frame, the impeller appears stationary and the wall of the tank moves in the opposite direction with a linear velocity, $\pi N T$, where $T$ is the tank diameter. The momentum equations can then be numerically solved, allowing for the additional Coriolis effects that result from the use of a non-inertial (accelerating) frame of reference. This method of using a single rotating frame is only valid for axisymmetric tank geometries, with no internal components and smooth walls. An example of such a simulation by Haque et al, (2006) is presented in the next section, where the effects of free surface modelling are also discussed.

For baffled vessels (or tanks containing other stationary internals), a similar approach may be employed using two grids, as is illustrated in Figure 0.12: (i) an inner grid that moves with the impeller in a rotating frame of reference, as described above and (ii) an outer grid that is stationary with respect to the walls and baffles, which are at rest. The inner grid is usually contained within a cylinder that surrounds the impeller; its radius should extend beyond the blade tips and outside the region where the trailing vortices are found, but not as far as the baffles; the height should be sufficient to contain the impeller and any features of the trailing vortices for axial and mixed flow impellers; see for example, Schafer et al. (1998). The momentum and continuity equations are solved on the inner grid using a rotating frame of 
reference, and on the outer grid, in a stationary frame of reference. The results at the interface from the inner grid become boundary conditions for the outer grid, and vice-versa. This approach can be extended to cases with multiple impellers, on different shafts, operated at different rotational speeds; one rotating reference frame would be required for each impeller.

There are two methods for passing information for the boundary conditions at the grid interface using either a sliding mesh (SM) or multiple reference frame (MRF) model. In the sliding mesh formulation, the inner grid rotates at the impeller speed and continually passes angle-dependent information to the outer stationary grid and vice-versa. This occurs as a sequence of steps, in which the transport equations are solved iteratively between the inner and outer grids until convergence is obtained; the inner grid then slides, or clicks to a new angular position and the iterative process starts once more, passing information between the two grids. In this way, the solution is fully time dependent and models the detailed motion of the impeller relative to the baffles and tank wall. The cells at the interface between the inner and outer grids do not always line up and hence interpolation is required during the passing of information - this is known as a non-conformal interface. The SM method requires a transient calculation and is the most detailed and accurate method of modelling the impeller rotation; it should be used when there are strong interactions between the baffles and the blade. However, SM simulations are very computationally demanding and typically it will take several (10-20) impeller revolutions for the solution to come to a periodically stationary state; a further $10-20$ rotations may be required to collect statistical data and so quite long transient calculations have to be performed. If only time-averaged flow quantities (e.g. flow number, power number, mixing time, see 8.4.3) are required then it is unnecessary 
to account for the periodic motion of the impeller blades and a simplified, steadystate model would suffice, which leads to the MRF method

In the multiple reference frame model, the inner mesh does not move relative to the outer mesh and so there is a steady-state transfer of information across the interface (Luo et al. 1994). Thus the impeller is only modelled at one position relative to the baffles, which is acceptable where the blade-baffle interactions are weak. This is often the case in stirred tank flows, where the periodic effects are only found in the vicinity of the impeller; the flow in the outside region is essentially steady (Khan et al. 2004). This approximation may be verified by noting the changes in the MRF simulations for two different impeller orientations relative to the baffles. There is a very significant saving in the computational overhead using the MRF model, since the simulation is now steady-state. Furthermore, interpolation across the interface can be avoided using a conformal interface between the inner and outer grids, so that the cells on either side match up.

The mixing plane model is a variant of the MRF method, in which information at the interface is azimuthally averaged before it is passed to the other grid zone. Any tangential variations in the flow variables are removed, so that effectively the average for all impeller blade positions is pass to the outer grid. Any asymmetry in the outer geometry, for example, due to dip pipes, or wall inlets and outlets, cannot be correctly taken into account using the mixing plane model and hence it has not found widespread applications in mixing tank simulations (Marshall and Bakker, 2004).

In summary, both the SM and MRF approaches may be used to construct a priori models of the flows generated by the impeller, without the need for experimental data as boundary conditions. Although the sliding mesh model gives 
the most accurate representation of the rotating impeller, it is too computationally expensive to use for most situations and there appears to be little benefit in applying it with a RANS turbulence model. In contrast, LES simulations are inherently unsteady and must be performed on $360^{\circ}$ flow domains (no cyclic boundaries) and require sliding mesh representations of the impeller. For these reasons LES computational times are very long and the method has yet to have significant impact on the routine design of large scale equipment; nevertheless, as described in sections 8.4 .5 to 8.4 .7 , LES is the most promising route to successful simulations. The MRF model is a simplification that ignores blade-baffle interactions to yield a steady-state simulation of the flow, but includes a detailed prediction of the flows around individual impeller blades. With sufficient grid resolution, details of the trailing vortices can be modelled accurately with MRF.

\subsubsection{Prediction of mixer performance characteristics}

One of the first stages in designing a stirred tank mixing operation is to calculate the required power input to obtain a given process result. For example, the specific power input per unit volume $\left(P / V\right.$ in $\left.\mathrm{W} / \mathrm{m}^{3}\right)$ determines the mixing time, the mass transfer coefficient and may determine the distribution of solids in a vessel. The impeller power draw can be characterised by a dimensionless power number,

$$
P o=\frac{P}{\rho N^{3} D^{5}}=f(R e, F r)
$$

which is a function of the Reynolds number defined in eq.(0.16) and the Froude number for unbaffled tanks, since the shape of the surface vortex is determined by gravitational acceleration, $g$. 


$$
F r=\frac{N^{2} D^{2}}{g}
$$

At high Reynolds numbers $\left(\operatorname{Re}>10^{4}\right)$ in baffled vessels, the power number is a constant. Empirical relations of the form of eq.(0.49), or constant values of $P o$ at high $R e$, are available for standard impeller types and geometries (e.g. Hemrajani and Tatterson, 2004), but they do not always extend to a wide range of diameters ratios, $D / T$, or clearance ratios $C / T$, or to new impeller designs. For these cases, the power number can be quite successfully predicted by CFD from a torque balance on either the shaft and impeller blades, or the tank wall and baffles. This torque, $\Gamma$, results from an integration of the shear stress and pressure differences acting on these surfaces and is related to the power input by

$$
P=2 \pi N \Gamma
$$

In principle, the power input could also be obtained from a volume integral of the turbulence kinetic energy dissipation rate, $\varepsilon$, which is predicted directly in RANS simulations and can be calculated from LES. There is also viscous dissipation due to the mean flow velocity gradients, but this term usually makes a negligible contribution to the overall power input in turbulent flows.

$$
P=\rho \int_{V} \varepsilon d V
$$

In practice, however, RANS models significantly under-predict the turbulence quantities and hence the total power input is not accurately obtained from eq.(0.52). In both laminar and turbulent mixer applications, eq.(0.51), is the preferred method to calculate power draw, from the integrated torque on the impeller and shaft. 
The flow number is a dimensionless measure of the strength of the discharge stream generated by the impeller. It is defined in terms of the volumetric flow rate, $Q$, obtained by integrating the velocity distribution over the parts of the swept surface through which the discharge stream passes. For example, for a Rushton disk turbine, this surface would be a cylinder, with diameter of just greater than the impeller diameter $D$ and a height just greater than the blade width $W$ (the region marked as in inlet boundary condition in Figure 0.11).

$$
F I=\frac{Q}{N D^{3}}=f(R e)
$$

For high Reynolds number flows, $F /$ is a constant whose value can be obtained by integrating velocity profiles, measured experimentally by LDA or PIV (see Chapter 7), or more straightforwardly from CFD simulations. If the surface for integration is moved away from the impeller swept surface, then larger flow numbers result, since they include the effects of entrainment into the impeller discharge stream. This type of flow number is a measure of the circulation flow inside the vessel, rather than the flow emanating from the impeller itself. The concept of the discharge flow gives a good qualitative indication of the impeller's ability to generate fluid motion, but is not particularly useful in the design of mixing systems, unless it can be directly linked to blend times or solids suspension criteria.

The dimensionless mixing time is another global characteristic of a stirred tank agitator that has been traditionally measured by experiment, but which is now potentially accessible by CFD simulation. In experimental studies, the mixing is quantified by the time required for the concentration fluctuations of a tracer material to have decayed to within $5 \%$ of the final well-mixed concentration (95\% mixing time, $\left.\theta_{95}\right)$. Salt or dye concentration are commonly used to mark the tracer and detection 
is then by conductivity probe, optical absorption or visual observation. The dimension mixing time is

$$
N \theta_{95}=f(R e)
$$

At high Reynolds numbers the dimensionless mixing time is a constant. There have been many attempts to calculate this mixing time, by solving the species transport equation, to predict the transient distribution of a tracer that has been added to a developed flow inside a stirred tank. These types of simulations are discussed further in section 8.4.6.

\subsubsection{Simulation of unbaffled or partially baffled stirred tanks}

For high Reynolds number, turbulent flows, most industrial mixing vessels would be equipped with a set of four wall baffles, which redirect the swirling flow generated by the impeller to produce axial and radial velocity components and improved top to bottom mixing. Glass-lined vessels are an exception and may only contain one or two beavertail baffles, which are suspended from the reactor head. Some mixing vessels used in food processing may also operate without baffles, because of CIP cleaning requirements. For low Reynolds number mixing operations (viscous systems) in the laminar or transitional regimes, internal obstructions may make mixing slower and hence vessels are often operated without baffles.

For unbaffled, or partially baffled, vessels the flow contains strong tangential velocity components and a surface vortex tends to form at all but the lowest impeller speeds. Hence the free surface is far from flat and it would be inappropriate to represent this boundary using a symmetry condition, as described in section 8.2.7.

The first studies of unbaffled vessels ignored the surface vortex feature of the flow (e.g. Armenante et al. (1997) who studied vessels with a lid), but more recent 
works have attempted to simulate the free surface shape using, for example using the volume of fluid (VOF) method first proposed by Hirt \& Nichols (1981). This is a form of two-phase simulation, which tracks the volume of fluid, $f$ using a transport equation. A single set of momentum and continuity equations are solved, for either the gas phase $(f=0)$, the liquid phase $(f=1)$, or the mixture $(0<f<1)$; mean physical and transport properties are calculated from the value of $f$. For no interphase mass transfer the VOF equation simplifies to

$$
\frac{\partial f}{\partial t}+(\boldsymbol{u} \cdot \nabla f)=0
$$

In practice, the change from gas to liquid is not abrupt and hence specialised methods have to be applied to sharpen and interpret the interface (e.g. Rudman, 1997). VOF methods can take surface tension effects at the interface into account, although typically they should not be important for stirred tank flows, since the Weber number (a ratio of inertial to surface tension effects) is often much greater than unity.

$$
W e=\frac{\rho N^{2} D^{3}}{\sigma}>>1
$$

Haque et al. (2006) implemented such a VOF model within ANSYS CFX-5.7 to simulate the flow in an unbaffled tank agitated by a Rushton disk turbine, which was modelled using a single rotating frame of reference (see section 8.4.2). Their simulations showed that both the shear stress transport (SST: a hybrid $k-\varepsilon / k-\omega$ formulation) and the Reynolds stress (RSM) turbulence models gave reasonable predictions of the mean velocity fields and the surface shape of the central vortex, when compared to experimental measurements and a simplified forced / free-vortex model by Nagata (1975). The SST model was shown to more successful than the 
standard $k-\varepsilon$ model in predicting the tangential velocity distribution, which is key to correct prediction of the surface vortex shape.

There are only a few studies to date of stirred vessels that are partially baffled by dip pipes, beavertails of finger baffles. Campolo et al. (2002) and Campolo and Soldati (2002) presented a comparison of the flows generated by a retreat curve impeller and a turbofoil turbine in an industrial scale vessel $\left(12.5 \mathrm{~m}^{3}\right)$ containing two beavertail baffles, over a range of Reynolds numbers in the transitional and turbulent regimes. A sliding mesh model was applied to take into account the interaction between the two baffles and the impeller blades. The standard $k-\varepsilon$ turbulence model was selected and the free surface was assumed to be flat, such that it could be modelled as a zero shear stress boundary condition. It could be argued that it would have been better to use a VOF model in this case, since free surface vortices have been observed in the wake of the beavertail baffles (Li et al., 2004). Campolo's results were successfully validated by comparison of the calculated power number against experimental measurements; The impeller performance was characterised by the flow number and the advantages and disadvantages of retrofitting the vessel with a turbofoil to replace the retreat curve impeller could be clearly identified in terms of the flows and circulations generated in the upper parts of the vessel. The simulations clearly showed that reducing the bottom clearance of the impeller produced a more radial discharge stream.

In a similar study, Li et al. (2004) simulated a retreat curve impeller with a single cylindrical baffle, using the SST turbulence model, but again with a flat free surface. Their mean velocity predictions were successfully validated at the 20 litre scale using LDA measurements. However, as is common with RANS models, the turbulence kinetic energy, and hence the dissipation rate, were significantly under- 
predicted by CFD. Li et al. (2005) took this validated model and examined scale up from tank volumes of 0.5 litre to 20 litre, to mimic the process development in pharmaceuticals manufacture. Their work suggested that the macro-mixing characteristics of lab scale vessels should be approximately the same as in an industrial scale reactor, so long as turbulent flow can be maintained.

\subsubsection{Simulation of single phase flow in baffled stirred tanks}

Many researchers (e.g. Ranade and Joshi, 1990; Harris et al., 1996; Brucato et al., 1998a; Patwardhan 2001; Jones et al., 2001; Jaworski and Zakrzewska, 2002; Aubin et al., 2004a and b; Ochieng and Onyango (2008)) have applied Reynolds averaged Navier-Stokes (RANS) based turbulence models (mainly $k-\varepsilon$ model) to simulate flow in a fully baffled stirred tank. Following comparisons with LDA or PIV measurements, these authors have generally found that CFD gives a satisfactory prediction of the axial and radial mean velocity components and flow patterns. However, the tangential velocity components are reported to be variously under- or over- predicted, whereas the turbulence kinetic energy, $k$, and the turbulence energy dissipation rate, $\varepsilon$, are under-predicted by RANS CFD models. Aubin et al. (2004a) carried out a detailed review of the recent literature and also made their own investigations of different impeller representations, turbulence models and discretisation schemes. She showed that changing the impeller representation from MRF to SM had only a small effect on the radial and axial velocity components near the impeller, which were in good agreement with LDA data, as shown in Figure 0.15. The difference from experimental values in the calculated circulation numbers was less than $10 \%$ and power numbers were predicted within $5 \%$ (from the torque on the blades and shaft). Also, the impeller model made no significant improvement to the tangential velocity or turbulence kinetic energy, $k$, predictions. First-order upwind 
discretisations were found to give the worst estimates of $k$, although even higherorder schemes were under-predictive. Similarly changing from the standard $k-\varepsilon$ to the RNG $k-\varepsilon$ or RSM models did not improve the turbulence predictions for $k$. Thus, although RANS models successfully predict mean flow patterns, their ability to predict $k$ (and by inference $\varepsilon$ ) is poor.

Figure 0.16 shows a comparison of the CFD simulation of a Rushton disk turbine ( $\mathrm{Ng}$ et al., 1998), using a SM and the standard $k-\varepsilon$ RANS turbulence model. Fine grid resolution around the blades (around 10 grid points across the blade width are required) and the use of the SM, allows the trailing vortices to be visualised in the wake of each turbine blade. The turbulence kinetic energy, $k$, is a maximum in the core of these vortices, but the comparison with LDA measurements in Figure 0.16 (b) shows that in the region $0.2<r / T<0.3, k$ is under-predicted by about $50 \%$, which is a common problem for RANS models. Despite this deficiency, the mean flow patterns and velocity components are rather well predicted in $\mathrm{Ng}$ et al.'s (1998) simulations.

Harris et al., (1996) and Jaworski and Zakrzewska (2002) have studied more advanced RANS models, such as the Reynolds stress model (RSM), but these also suffer from similar difficulties in predicting the turbulence quantities. Armenante et al. (1997) reported good predictions of the mean velocities and turbulence using an algebraic stress model (ASM) but their simulations used a black box approach (see section 8.4.2), and cannot be considered an a priori calculation. The ASM is a simplification of the RSM, which proposes a set of approximate algebraic equations which relate the Reynolds stresses. Other than the study by Armenante et al. (1997), the ASM model has not been extensively tested and validated for prediction of turbulent flows in stirred tanks. 
It is well known from the literature that large eddy simulation (LES) can predict excellently the time-averaged mean and turbulence flows (Derksen, 2001; Derksen and Van den Akker, 1999; Revstedt et al. 1998, Yeoh et al., 2004; Hartmann et al., 2004; Li et al., 2007; Jahoda et al., 2007; Tyagi et al., 2007; Yapici et al., 2008). LES is a three-dimensional numerical simulation of turbulent flow where large eddies are resolved and the effects of subgrid-scale eddies, which are more universal in nature, are modelled. An example of two flow snapshots are shown in Figure 0.17 ; the instantaneous structure of the trailing vortex system behind each blade is clearly visible, as is the interaction of the discharge stream with the baffle. LES to some extent tends to over-predict the turbulent kinetic energy (Hartmann et al., 2004) but generally it predicts turbulent flows much better than RANS models. However, full LES simulations are still very expensive to solve. For example Alcamo et al. (2005) needed 33 days to solve a LES of an unbaffled tank on a Pentium IV $3 \mathrm{GHz}$ computer. Earlier Derksen (2001) reported that it took a month (up to 44 hours per impeller revolutions) to solve the LES of a baffled stirred tank on a cluster of four parallel processors.

It is also possible to solve the turbulence flow in stirred tanks directly using the exact Navier-Stokes equations without any modelling, but this requires a grid and time step that resolves even the smallest eddies. Such a method is known as a Direct Numerical Simulation (DNS). Recently, DNS has been applied to predict the turbulence flows in a stirred tank by Verzicco et al. (2004) and Sbrizzai et al. (2006). These authors concluded that DNS predicts the turbulence related quantities such as turbulent kinetic energy and turbulent energy dissipation rate much better than RANS model. However, both works only involved a low Reynolds $\operatorname{Re}=1636$ (a 
transitional flow) in an unbaffled tank, suggesting that DNS for a baffled stirred tank at high Reynolds number is still far from the reach of current computer resources.

\subsubsection{Mixing and blending simulations}

Accurate prediction of mean velocity and turbulence $k$ and $\varepsilon$ fields is only the first stage in using CFD as a practical design tool for mixing operations that involve species transport, chemical reaction and phase dispersion. Clearly, the deficiencies of RANS models in predicting the energy dissipation rate in single phase flows is likely to impact on the simulation of these more complex processes. As an example, for blending operations, a standard correlation for the $95 \%$ batch mixing time is (Ruszkowski, 1994)

$$
\theta_{95}=5.91 \varepsilon^{-1 / 3}\left(\frac{T}{D}\right)^{1 / 3} T^{2 / 3}
$$

which suggest the importance of at least the mean dissipation rate (Nienow, 1997). In gas-liquid or liquid-liquid dispersion, the breakage kinetics and equilibrium bubble (droplet) size are related to the maximum local dissipation rate.

Ochieng and Onyango (2008) demonstrated the difficulties in predicting the 95\% mixing time from CFD simulations using a standard $k-\varepsilon$ RANS model, applying the SM method. The blending process was simulated by solving eq.(0.31) to follow the transient concentration variations of a tracer that had been released as a point source in the flow. The mixing time was calculated from the concentration variations at a single point in the flow, as described in section 8.4.3 and was compared to (i) experimental measurements based on conductivity and decolourisation of a reactive indicator and (ii) empirical correlations, such as eq.(0.57). The measured mixing times and those predicted by literature correlations agreed to within $10 \%$. In 
contrast, the CFD predicted times were around $100 \%$ longer, despite the fact that the mean velocity fields agreed well with LDA measurements. Nevertheless Ochieng and Onyango (2008) were able to deduce the qualitative effects that reducing the impeller off bottom clearance gave shorter mixing times.

Bujalski et al. (2002) performed mixing simulations of Rushton turbine, using a $k-\varepsilon$ RANS model and found that the mixing time was very sensitive to the tracer concentration monitoring point; in contrast, experimental $\theta_{95}$ measurements do not show the same level of sensitivity to probe location in fully turbulent flows. For many of their monitoring locations the mixing times were significantly over-predicted by a factor of about 2. Montante et al. (2004) were able to make satisfactory predictions of $\theta_{95}$ using a RANS based CFD model, for a multiple impeller system, but were only able to do so by adjusting the value of the turbulent Schmidt number in eq.(0.31). For many turbulent flows, a value of $S c_{T}=0.7$ is applied, but Yimer et al. (2002) have reported that improvements in the concentration field predictions can be obtained using lower values. Montante et al. (2004) had to reduce the turbulent Schmidt number to an exceptionally low value, $S c_{T}=0.1$ to match the experimental mixing times. Examination of eq.(0.32) shows that reducing $S c_{T}$ increases the eddy diffusivity and hence becomes an ad hoc correction to the under-prediction of the turbulence quantities, $k$ and $\varepsilon$. The generality of this approach for stirred tank flows has not yet been demonstrated.

Yeoh et al. (2005) used an LES turbulence model to predict tracer dispersion and mixing times in a vessel agitated by a Rushton turbine and obtained very good agreement with experimental values of $\theta_{95}$ and estimates obtained from literature correlations. They attributed this success to the fact that their LES generated flow 
fields were very well validated in terms of the mean and rms fluctuating velocity distributions. The latter, of course, are related to the turbulent diffusivity of the tracer. Notably, their implementation of the species transport eq.(0.31) used $S c_{T}=0.8$, which is close to the default value for turbulent shear flows. Their results support the conclusion that the main problem with RANS simulations of mixing processes is that the turbulence quantities are under-predicted. A further problem associated with mixing time calculations is numerical diffusion, which results from inaccurate discretisation methods and has a strong effect on the solution of the species transport equation, smearing out concentration gradients more quickly than would be expected by laminar diffusion alone.

\subsubsection{Multi-phase simulations}

As noted in section 8.4.1, there are many industrial examples of multi-phase mixing operations in the chemical, biochemical and food industries. In principle, multi-phase systems also can be simulated using the techniques of CFD, although clearly the physics of these flows is considerably more complicated. The discussion of section 8.4.4 described one technique for dealing the two-phase (gas-liquid) flows using the VOF approach. This is suitable for cases where each phase can be treated as continuous, with an identifiable and fairly simple shape of interface: the previous example in section 8.4.4, was of an unbaffled vessel with a free surface vortex. However, for phase dispersion and suspension operations, there will be well defined dispersed and continuous phases, with many interfaces, and the VOF method would be quite impractical. Other approaches are described below.

At low volume fractions $(<10 \%)$ of dispersed phase, it is possible to perform Eulerian-Lagrangian particle tracking calculations. The background continuous fluid flow is obtained by solving the single phase (Eulerian) equations, as previously 
described in section 8.2; a Lagrangian equation of motion for the particles is then formulated, for example (Rielly and Marquis, 2001)

$$
\begin{aligned}
\boldsymbol{F}=\rho_{p} \frac{D_{p} \boldsymbol{v}}{D t}= & \rho_{f} \frac{D_{f} \boldsymbol{u}}{D t}-\rho_{l} C_{M}\left(\frac{D_{p} \boldsymbol{v}}{D t}-\frac{D_{f} \boldsymbol{u}}{D t}\right)-\rho_{f} C_{L}(\boldsymbol{v}-\boldsymbol{u}) \times \boldsymbol{\omega} \\
& -\frac{3}{4} \frac{\rho_{f} C_{D}}{d_{p}}(\boldsymbol{v}-\boldsymbol{u})|\boldsymbol{v}-\boldsymbol{u}|-\left(\rho_{p}-\rho_{f}\right) \boldsymbol{g}
\end{aligned}
$$

which relates the particle velocity vector, $\boldsymbol{v}$ to the fluid velocity vector $\boldsymbol{u}$ and includes effects such as the pressure gradient, added mass effect and lift, drag and buoyancy forces. This equation can be integrated through time to yield individual particle tracks; the stochastic effects of particle interactions with eddies can also be included, as is shown in Figure 0.18.

By following a large number of particles, the dispersed phase volume fraction distributions can be deduced, as well as time histories for how the particles sample, the turbulence and strain rate fields. Figure 0.18 shows how inhomogeneous the flow environment is in a stirred vessel, with very large peaks in the dissipation and strain rate as the particle passes through the impeller region.

Commercial software is also able to implement two-way coupling between continuous and dispersed phase flows: Eulerian continuous phase and Lagrangian discrete phase are run alternately, until the momentum exchanges are converged. The method is limited to low volume fractions, because it does not include the effects of momentum exchanges through particle-particle collisions. If the flows involved heat and mass transfer between the dispersed and continuous phases, then these effects can be included in the same way as inter-phase momentum exchange.

For higher volume fractions, it is less time consuming and better to use an Eulerian-Eulerian two fluid model. Both the dispersed and continuous phases are assumed to behave as interpenetrating continua, represented locally by their phase 
volume fractions. The continuity and momentum equations are then written separately for each phase and additional terms are included for the effects of momentum exchange (predominantly drag) between the two-phases (Elgobashi and Abou-Arab, T. W.,1983). A similar approach can be applied to the energy and species transport equations, except that now the exchange terms represent heat or mass transfer between the phases. The implementation of RANS based turbulence models is much more complex in multi-phase flows: the averaging of the momentum equations for each phase produces a much larger number of unknowns (in the single phase case, the six Reynolds stresses are the only unknowns generated by the averaging process), which have to be modelled empirically (Simonin and Viollet, 1990). Furthermore, although there is no restriction on the range of dispersed phase volume fractions that can be modelled, the effects of particle-particle collisions are not included — in Eulerian granular multi-phase models, (e.g. Ding and Gidasapow, 1990), these effects can be included through a solids pressure term.

Solids suspension in a liquid-phase is probably the easiest mixing operation to simulate, given that typically both size and volume fraction of the dispersed phase will be known; in contrast, in gas-liquid flows the volume fraction and bubble size are not known at the outset of the calculation and hence this represents the most challenging case. Montante and Magelli (2005) carried out a thorough study of CFD modelling methods to predict the solids distribution in tanks agitated by multiple Rushton turbines and pitched blade impellers. They concluded that dilute solid-liquid systems $(<6 \% \mathrm{v} / \mathrm{v})$ can be fairly well predicted, so long as (i) the single phase mean velocity field is accurate and (ii) the drag coefficient is corrected to allow for the effects of turbulence (Brucato et al., 1998b). They recommended the use of the mixture turbulence model in which the two phases are assumed to share the same $k$ 
and $\varepsilon$. In addition, there were some minor improvements for higher volume fractions when the effects of particle-particle interaction were included through the granular multi-phase model. It should be noted that all such solid-liquid CFD simulations need to be conducted above the just-suspended speed of the particles (Zwietering, 1958), since the physics of particle settling, saltation and resuspension which occur in the boundary layer at the base of the tank, are not included in the model.

As has been noted previously, LES models are much better equipped to simulate the turbulence in a stirred tank flow, but at very considerable computational cost. This has deterred all but a few from performing solid-liquid calculations based on LES. An exception is presented by Derksen (2003), who simulated a $1 \% \mathrm{v} / \mathrm{v}$ fraction of $300 \mu \mathrm{m}$ diameter particles agitated by a Rushton disk turbine. The liquid flow was obtained using LES on a grid of some 14 million cells; Lagrangian tracking of almost 7 million particles was carried out over 10-20 impeller revolutions, with 2800 time steps per revolution and both two-way coupling and particle-particle interactions were included; importantly the latter were required to keep the particles in suspension, even though the impeller speed was above the just-suspended condition. Guha et al. (2008) recently presented a comparison between (i) solidliquid CFD simulations based on Eulerian-Eulerian RANS models and Derksen's (2003) LES particle tracking and (ii) experimental measurements from Computer Automated Radioactive Particle Tracking (CARPT). LES was shown to improve the prediction of fluid tangential mean velocities, but there were significant discrepancies in the particle velocities at the impeller level. Slip velocities from the EulerianEulerian model were very much smaller those obtained via LES, which is rather significant, since the inter-phase momentum exchange (drag) depends directly on this quantity. Despite this finding the solids concentrations at the impeller level were 
broadly similar for both CFD models, with the LES predicting slightly lower values. Thus although solid-liquid simulations can be conducted, there are still a number of uncertainties related to the physics of these flows, particularly with respect to particle-particle and particle-impeller interactions. There are even issues about the calculation of the drag coefficient for such flows: Montante and Magelli (2005) recommended correction of the drag coefficient to allow for local turbulence levels (Brucato et al., 1998b), but not for local solids volume fraction (Crowe et al., 1998), whereas Derksen (2003) makes the opposite recommendation, which seems to be the more conventional thinking.

Simulations of gas-liquid flows are more complex still, because the bubble size is not known a priori and results from a balance of breakage and coalescence events, depending on local energy dissipation rates. The first gas-liquid simulations assumed a constant bubble size, based on either experimental evidence or chosen as a fitting parameter to match the gas hold distribution or velocity measurements.. They then proceeded to formulate the problem in a very similar way to the solidliquid case, sometimes even using the same drag laws as for solid particles! For example, Montante et al (2007), chose a bubble size of around 1-2 mm diameter with a terminal velocity of around $0.12 \mathrm{~m} / \mathrm{s}$ (quite small for an agitated air-water flow) and successfully validated their predicted liquid phase velocity distributions against two-phase PIV measurements. The experimental mean gas volume fraction of about $1.6 \%$ was also predicted by CFD, but distributions of local holdups were not available for comparison.

As shown experimentally by Barigou and Greaves (1992) and Laakkonen et al. $(2005,2007)$ the distribution of bubble sizes varies inside the stirred tank depending on the spatial position. Generally, bubble sizes around the impeller 
discharge stream are the smallest due to breakage caused by the high turbulence dissipation rates. Knowledge of bubble sizes is necessary in a two-phase CFD model since they affect the momentum exchange through drag. In addition, local bubble sizes and the local gas volume fraction are required for the calculation of the interfacial area, which is an important variable in designing an aerated stirred tank to achieve a required rate of gas-liquid mass transfer. Relaxing the assumption of a constant and uniform bubble size, requires a new level of sophistication in CFD modelling, to account for the dynamics of breakage and coalescence events. A rigorous approach would be to couple the two-phase fluid dynamics simulations, for example using an Eulerian-Eulerian continuum model, with a population balance equation (PBE) to represent the evolution of the bubble size distribution. The solution of the PBE is not a trivial task, since it requires detailed knowledge of kinetics for the breakage and coalescence events and for the distribution of bubbles that result from each breakage. Equation (0.59) shows how the local bubble size distribution by number, $n(L)$, evolves in time; in solving such an equation, $n(L)$ has to be represented as a set of discrete size classes or by the moments of the distribution. Either approach results in a set of equations derived from eq.(0.59), which have to be solved for every computational cell in the flow domain.

$$
\begin{aligned}
\frac{\partial(n(L))}{\partial t} & =\underbrace{\int_{L}^{\infty} \beta(L, \lambda) g(\lambda) n(\lambda) \mathrm{d} \lambda}_{\text {birth due to breakage }}+\underbrace{\frac{L^{2}}{2} \int_{0}^{L} \frac{F\left(\left(L^{3}-\lambda^{3}\right)^{1 / 3}, \lambda\right) n\left(\left(L^{3}+\lambda^{3}\right)^{1 / 3}\right) n(\lambda)}{\left(L^{3}-\lambda^{3}\right)^{2 / 3}} \mathrm{~d} \lambda}_{\text {birth due to coalescence }} \\
& -\underbrace{g(L) n(L)}_{\text {death due to breakage }}-\underbrace{n(L) \int_{0}^{\infty} F(L, \lambda) n(\lambda) \mathrm{d} \lambda}_{\text {death due to coalescence }}
\end{aligned}
$$

The results for the mean bubble size, $d_{32}$, from the PBE are passed back to the fluid flow calculation and affect the drag between the phases; the kinetic terms, $\beta(L, \lambda)$, 
$g(L)$ and $F(L, \lambda)$ are all related to the local hydrodynamics, mainly through the dissipation rate, $\varepsilon$. Thus the Eulerian-Eulerian two-fluid calculation is coupled to the PBE solution. This type of approach is still in its infancy, although some promising results have been generated so far, by workers such as Montante et al. (2008), Laakonen et al. (2007) and Gimbun et al (2009). Figure 0.19 shows some example results from Laakonen et al. (2007) for the gas holdup and bubble size distributions; the latter are rather far from uniform and the distribution of bubble sizes has a significant effect on the local mass transfer coefficient and interfacial area.

Thus very significant advances have been made in recent years in developing better and more realistic multi-phase models, yet they come with a substantial computational overhead and are not yet usable for industrial design purposes. A coupled CFD-PBE solution inevitably requires the application of RANS turbulence models to limit the computational cost of the velocity field calculations, yet these are known to suffer from deficiencies in their ability to predict the dissipation rate. Unfortunately this is precisely the quantity that determines the local kinetics within the PBE, which makes this an exciting area for future developments.

\subsection{Application to food mixing operations}

\subsubsection{Challenges for simulation of food processes}

Figure 0.1 showed that CFD has been increasingly applied within the food industries and Sun (2007) presents a very wide range of examples in his book "Computational Dynamics in Food Processing". However, there are relatively few literature publications related to food mixing, whereas in contrast, there are numerous CFD studies of a wide range of mixing unit operations in chemical and biochemical 
engineering. Several of these have been described and discussed in previous sections. So, what has held back the application of CFD to food mixing processes? The following paragraphs provide a personal view of the challenges in applying CFD in this area.

Knowledge of physical and transport properties: Many food materials are complex mixtures, containing a structure which is irreversibly (occasionally reversibly) affected by shear and elongation, leading to non-Newtonian behaviour and possibly elastic effects. Standard commercial software allows the treatment of shear-thinning and yield stress materials, but more complex visco-elastic behaviour (typical of pastes and doughs) is much more difficult to characterise experimentally and requires more specialised numerical approaches, e.g. through the add-on module to POLYFLOW. Doughs, for example, are mixed to align proteins and develop structure; deformation coupled to chemistry results in a material that changes its rheology and properties during mixing, which is a real difficulty in fluid flow modelling. Other pastes and dispersion that thicken due to mixing and heat transfer are also problematic to simulate. Where chemical reactions occur in food mixtures, their specific rate laws are often unknown and hence it may be impossible to include important chemistry within the simulation.

Transitional flows: the vast majority of the flows discussed in section 8.4 , were in the fully turbulent flow regime. But with viscous fluids, such as are common in food processes, it is likely that the flow is transitional, in the range $10<R e<10^{4}$, or even that the Reynolds number changes during mixing, due to heat transfer and / or thickening. Some low Re turbulence models are better suited to weakly turbulent flows, but there has not really been enough research to assess their applicability in transitional cases (the stirred tank mixer is unusual in that the range of transitional 
flow is rather large, and it is not uncommon to find a turbulent type flow close to the blade tips, which re-laminarises in the bulk of the tank).

Complex mixer geometries: the standard representations of the impeller for a cylindrical stirred vessel were discussed in section 8.4.2. The best of these techniques, SM and MRF, require that the rotating blades are enclosed in a simple surface, which is usually a volume of revolution. Industrial dough mixers and planetary mixers involve intermeshing blades, for which there is no way of defining non-overlapping moving reference frames. Therefore, remeshing, adaptive meshs or superposition techniques are required, and typically these will only be available in bespoke or specialist software. Even then, simplifications to the mixer geometry may be required to make the simulation tractable. Some further examples of these specialist techniques are given in section 8.5.2.

High volume fraction multi-phase mixtures: economic optimisation of process equipment generally requires intensive operation at high dispersed phase fraction, which maximises specific kinetic rates (e.g. of mass transfer or chemical reaction). The Lagrangian tracking technique of section 8.4.7 is restricted to low volume fractions $(<10 \%)$, although with some additional effort, two-way coupling can be included; inclusion of particle-particle collisions involves simultaneous tracking of the particles, which makes this technique infeasible for highly concentrated dispersions. Although the Eulerian-Eulerian methods (see section 8.4.7) are more easily implemented for high volume fractions, they do not include particle-particle collision effects. Therefore simulation of important processes, e.g. secondary nucleation by crystal-crystal or crystal-impeller collisions is not yet possible at high solids fractions. 
Realistic simulations of industrial food mixing operations may involve one or more of the difficulties discussed above, but nevertheless, some progress can be made by using simplifying assumptions which can be tested out against experimental evidence. The approach really needs to be that doing something is better than doing nothing! The following sections gives a selection of recent examples, where researchers have been pushing the boundaries to ever more complicated simulations relevant to food mixing operations.

\subsubsection{Examples of food applications}

As noted in section 8.1.1, despite the ubiquitous use of CFD for the analysis of food processes, there are relatively few examples of applications to food mixing operations. Most of the applications are related to homogenisations, bioreactors, crystallization and modelling of viscous fluid mixing. Commercial CFD code without modification are often not capable of dealing with the complex fluid flow of food mixtures, especially when it related to viscous fluid and particle / bubble dynamics. Connelly and Kokini (2007) provide a recent review, which highlighted advances in only three areas related to food: (i) high viscosity non-Newtonian blending; (ii) visco-elastic dough mixing in simplified mixer geometries and (iii) crystallisation and nano-particle production. These are amongst the first attempts at addressing some of the issues raised in section 8.5.1.

CFD has been applied to model two-phase flow in bioreactors for shearthinning xanthan fermentations by Venneker et al. (2002) and Laakkonen et al. (2006). Venneker implemented a one-way coupled PBE to model a lab scale bioreactor and found satisfactory predictions for the local gas hold-up, except around the impeller region. Laakkonen employed a coupled CFD-PBE approach for modelling the mass transfer in a lab and pilot scale bioreactors. They obtained good 
predictions of the local bubble size, but only by adjusting the constants within the breakage and coalescence kernel. This practice is essentially empirical and is not fully predictive for cases where the experimental data is not available. They also reported a satisfactory prediction of the mass transfer coefficient.

Tanguy's group at the Ecole Polytechnique de Montreal have for many years studied finite element simulations of low Reynolds number flows in helical ribbon and co-rotating screw extruders. These flow geometries are characterised by narrow gaps and one or more moving parts which rotate relative to each other and hence advanced meshing techniques are required. Giguere et al. (2006) reviewed the recent history of finite-volume or finite element methods to deal with complex moving geometries. Quasi steady simulations on a number of meshes, which represent the geometries at each time step, are feasible but cumbersome to implement; of order 100 separate meshes may be required to model the impeller throughout the period of its motion. Improved strategies include: (i) allowing the mesh to deform between time steps and only remeshing highly skewed or high aspect ratio elements; (ii) the mesh superposition technique (MST) which involves combining a static and dynamic mesh (Avalosse et al, 2002) and is implemented in the commercial solver POLYFLOW; and (iii) the fictitious domain method by Bertrand et al. (1997), which is similar to MST, but enforces the kinematics of the moving boundary in a different way.

Giguere et al. (2006) applied the fictitious domain method, coupled with a mesh refinement strategy to a helical ribbon mixer and obtained satisfactory agreement with experimentally measured power inputs for a Newtonian liquid. Iranshahi et al. (2006) included a mixing time calculation within their study of a Paravisc impeller (a hybrid of an anchor and a double helical ribbon). As noted in section 8.4.6, solution of the species transport equation is problematic and prone to 
numerical diffusion effects. An alternative way to represent mixing is by particle tracking and trajectory analysis of a large number of mass-less particles which are released from a single point and then advected in the flow. A mixing time can then be evaluated from the decay of the intensity of segregation, which is related to spatial variance of the concentration of particles. In Iranshahi et al.'s (2006) work, the predicted mixing times were within $6 \%$ of experimentally measured values and the method allowed the mixing efficiency of the Paravisc to be compared with more conventional low Reynolds number impeller types.

Fictitious domain and MST impeller representation methods have also been applied to the simulation of dough mixing operations. The first attempts, e.g. by Jongen et al. (2000) assumed Newtonian behaviour in a simplified 2D geometry, but were still able to assess the different relative amounts of rotation and shear and elongational deformation that were imposed on the dough during mixing. These deformations have an important effect on the alignment of proteins inside the dough and hence on the development of the visco-elastic structure which is so important in determining the texture of the baked loaf. Connelly and Kokini (2004) took this further by examining the effects of the fluid rheological properties (Newtonian, shearthinning inelastic, constant viscosity linear visco-elastic and non-linear visco-elastic fluids) on the deformation rate fields generated in a simplified 2D model of a dough mixer, modelled using a rotating frame of reference. The streamline contours and flow pattern were similar for each rheology, but the choice of constitutive equation led to differences in the deformation rates, particularly in the narrow gap between the moving blade and the walls. The amount of elongational flow, important for structure development, was found to depend on the constitutive model. Nevertheless, the 
results indicated regions of the device where mixing was poor and deformation rates were low.

Connelly and Kokini (2006a) extended their previous 2D work to a more realistic 3D geometry, namely a simplified version of the Farinograph, which is a sigma-blade used for characterisation of flours and for determining water absorption. The mixer features two, non-intermeshing sigma blades, as shown in Figure 0.20. MST was applied to mesh around the moving blades and both Newtonian and shearthinning fluids were examined. Although the flow patterns were in good agreement with LDA data, the predicted shear rates were an order of magnitude too low when compared to experimental measurements at similar positions in the flow. Poor refinement of the meshing technique close to the blades was one reason for this underestimate. Connelly and Kokini (2006b) performed further CFD mixing studies in the Farinograph geometry, based on tracking of $10^{4}$ particles that were initially segregated in the two halves of the mixer. Figure 0.21 (a) shows the initial configuration and (b) the situation after 3 cycles; around the blades, the particles appear more homogeneous, but near the top of the Farinograph, the mixture remains highly segregated. So although CFD simulations have the capabilities to model the flows, deformation and mixing rates in these complex geometries, more work still is required before they can be used for thorough analysis of the mixing performance. A further simplification used in Connelly and Kokini's studies is that the mixer runs full of fluid. In practice, however, dough mixers seldom run full, and the dough itself is thrown around the mixer, resulting in a very complex free surface flow. Treatment of this type of dynamic free surface flow is beyond current capabilities. 
Some food processes involve a crystallisation stage. Indeed simulation of crystallisation processes and in particular the prediction and control of the crystal size distribution is a subject of much current research. Population balance equations, equivalent to eq.(0.59), but including the additional effects of nucleation and crystal growth, have to be solved in conjunction with the equations describing the hydrodynamics to predict the evolution of the crystal sizes in a batch vessel. In addition, the supersaturation, which provides the driving force for nucleation and growth, may result from chemical reaction, cooling, or anti-solvent addition. The supersaturation is likely to be non-uniformly distributed in space, but it in principle it can be calculated by solving energy and species transport equations in addition to the fluid flow modelling. Thus, these processes are amongst the most complicated that have been studied by CFD methods. One simplification that can be used is that the crystals are often small, so that they follow the fluid motion and hence only single-phase flow calculations are necessary.

Wei et al. (2001) applied a moment-based population balance (based on nucleation and growth only) to predict the mean and standard deviation of the crystal size distribution produced from a semi-batch precipitation of barium sulphate $\left(\mathrm{Ba}_{2} \mathrm{SO}_{4}\right.$ is a much studied model system, due to detailed knowledge of its kinetics and thermodynamics). Their predicted Sauter mean sizes compared well to experimental measurements, when both the feed time and impeller speed were varied, demonstrating that such studies have the potential to design and optimise semi-batch reactive crystallisations. However, as Rielly and Marquis (2001) have pointed out, reliable and detailed knowledge of the local kinetics of reaction, growth, nucleation and agglomeration is often missing for systems of practical or commercial importance. There is much debate about the suitable forms for kinetic rate laws. 
even for something as simple as growth — external mass transfer and surface reaction effects can lead to a variety of rate laws which depend on the local supersaturation and crystal size.

The moment transport equations used by Wei et al. (2001) run into closure problems for processes that involve agglomeration or crystal breakage. A more advanced method, the quadrature method of moments (QMOM) replaces each of the integral expressions for the moments by a quadrature approximation using a small number of weights and abscissas. Marchisio et al. (2003) described how QMOM may be applied to systems that exhibit agglomeration and breakage, using simplified kernels. Later, Gavi et al. (2008) successfully applied QMOM, coupled with micromixing model and a RANS CFD model to predict the size of nano-agglomerates of $\mathrm{Ba}_{2} \mathrm{SO}_{4}$ formed in an impinging jet micro-reactor. Comparisons with experiments were favourable (at the low barium excesses used), but required further testing for conditions where agglomeration is more likely to occur.

\subsection{Closing remarks}

This chapter has outlined the procedures for approaching CFD simulation and has discussed the underlying equations and numerical methods which are built into commercial packages. The more specialised methods for simulating stirred tank flows are also described and applications to single phase flow simulations are discussed. More complex methods to deal with multi-component mixing situations and multi-phase flows are presented and examples from the literature have been analysed. The intention has been to emphasise at each stage the need for a good fundamental understanding of fluid mechanics and transport processes, so that justified decisions are made about the simplifying modelling assumptions, 
approximations and boundary conditions. Commercial CFD packages have been made much more user-friendly in recent years, but they are not intended for the novice who has little or no knowledge of fluid mechanics.

Very significant advances have been made in the last 30 years in the numerical solution of the equations of fluid motion using CFD methods and yet there are still a very large number of problems to be solved to make it an easily accessible tool which is routinely applied for design and optimisation in food mixing. The final section of this chapter discussed some of the barriers to the application of CFD to realistic mixing processes used in the food and allied industries, but it also looked at some recent studies which aimed to address at least some of these issues. In many cases, it is not simply the lack of computing power which is the problem-admittedly, turbulent flows modelled with LES or DNS require very significant resources—but rather that there are real difficulties in characterising the evolving non-Newtonian multi-phase behaviour of many food products, representing the complex geometries of some food mixers, dealing with unknown chemistry or incomplete knowledge of reaction rates and simulating high volume fraction dispersed phases or complex free surface shapes. The accuracy of any prediction is only as good as the underlying chemistry and physics and hence as knowledge improves in these areas it is expected that CFD will emerge as a useful tool for food applications, in just the same way that it has already been accepted in other engineering fields. 


\section{Notation}

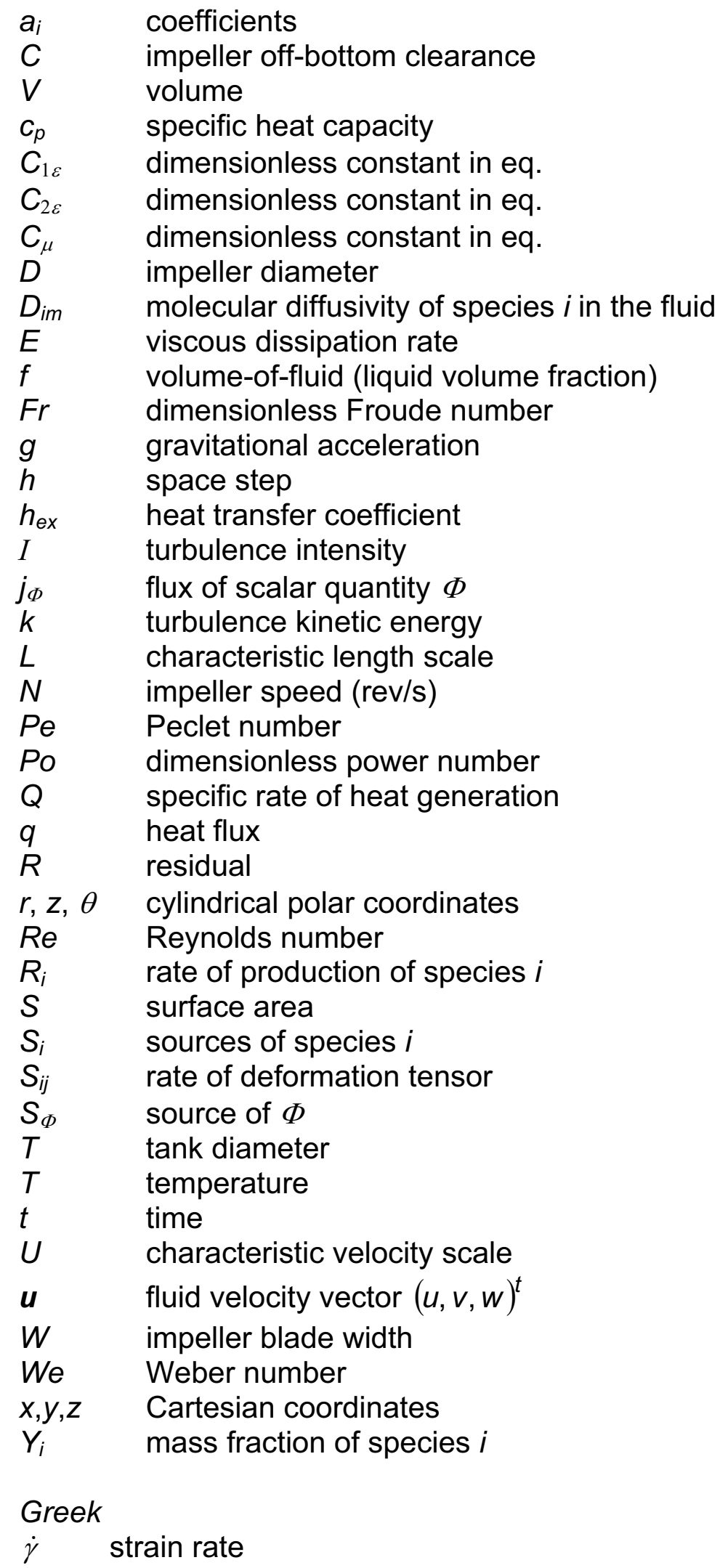


$\Phi \quad$ a general scalar quantity

$\Phi^{\prime} \quad$ fluctuating value of $\Phi$

$\bar{\Phi} \quad$ mean value of $\Phi$

$\Gamma \quad$ diffusivity of scalar $\Phi$

$\Lambda \quad$ integral length scale

$\varepsilon \quad$ turbulence energy dissipation rate

$\kappa \quad$ thermal conductivity

$\mu \quad$ fluid viscosity

$\rho \quad$ fluid density

$\sigma \quad$ surface tension

$\sigma_{k} \quad$ dimensionless constant in eq.

$\sigma_{\varepsilon} \quad$ dimensionless constant in eq.

$\tau_{i j} \quad$ stress tensor

\section{Subscripts}

ex external

in inlet

L laminar

$n$ normal

$T \quad$ turbulent

w wall 


\section{References}

Alcamo, R., Micale, G., Grisafi, F., Brucato, A., Ciofalo, M., (2005), "Large-eddy simulation of turbulent flow in an unbaffled stirred tank driven by a Rushton turbine", Chem. Eng. Sci., 60, pp. 2303-2316.

Armenante, P.M., Luo, C., Chou, C. Fort, I., Medek, J., (1997), "Velocity profiles in a closed, unbaffled vessel: Comparison between experimental LDV data and numerical CFD predictions", Chem. Eng. Sci., 52, pp. 3483-3492.

Aubin, J., Fletcher, D.F., Xuereb, C., (2004a), "Modelling turbulent flow in stirred tanks with CFD: The influence of the modelling approach, turbulence model and numerical scheme", Exp. Therm. Fluid Sci., 28, pp. 431-445.

Aubin, J., Le Sauze, N., Bertrand, J., Fletcher, D.F., Xuereb, C., (2004b), "PIV measurements of flow in an aerated tank stirred by a down- and an up-pumping axial flow impeller", Exp. Therm Fluid Sci., 28, pp.447-456.

Avalosse, T., Rubin, Y., \& Fondin, L. (2002). "Non-isothermal modeling of corotating and contra-rotating twin screw extruders", J. Reinforced Plastics and Composites, 21, 419-429.

Barigou, M, Greaves, M, (1992), "Bubble-size distributions in a mechanically agitated gas-liquid contactor", Chem. Eng. Sci., 47, pp.2009-2025.

Batchelor, G.K. (2000), "An Introduction to Fluid Dynamics", Cambridge University Press, Cambridge, UK.

Bertrand, F., Tanguy, P.A. and Thibault, F., (1997), "Three Dimensional Fictitious Domain Method for Incompressible Fluid Flow Problems", Int. J. Numerical methods in Fluids, 25(6), 719-736

Bird, R.B, Stewart, W.E, Lightfoot, E.N (2007), Transport Phenomena, John Wiley \& Sons, Inc, New York, NY.

Brucato, A., Ciofalo, M., Grisafi, F., Micale, G., (1998a), "Numerical prediction of flow fields in baffled stirred vessels: A comparison of alternative modelling approaches", Chem. Eng. Sci., 53, pp. 3653-3684.

Brucato, A., Grisafi, F. and Montante, G., (1998b), "Particle drag coefficients in turbulent fluids", Chem. Eng. Sci., 53, 3295-3314. 
Bujalski, J.M., Jaworski, Z. Bujalski, W. and Nienow, A.W., (2002), " The influence of the addition position of a tracer on CFD simulated mixing times in a vessel agitated by a Rushton turbine", Chem. Eng. Res. Des., 80, 824-831

Campolo, M., Paglianti A., Soldati, A. (2002), "Fluid Dynamic Efficiency and Scale-up of a Retreated Blade Impeller CSTRs", Ind. Eng. Chem. Res., 41, pp.164-172.

Campolo M., Soldati, A. (2002), "Appraisal and Fluid Dynamic Efficiency of

Retreated-Blade and Turbofoil Impellers in Industrial-Size CSTRs", Ind. Eng. Chem. Res., 41, pp.1370-1377.

Connelly, R.K. and Cokini, J.L., (2004), "The effect of shear thinning and differential viscoelasticity on mixing in a model 2D mixer as determined using FEM with particle tracking", J. Non-Newtonian Fluid Mech. 123, 1-17.

Connelly, R.K. and Cokini, J.L., (2006a), "3D Numerical Simulation of the Flow of Viscous Newtonian and Shear Thinning Fluids in a Twin Sigma Blade Mixer", A.I.Ch.E.J., 52(10), 3383-3393.

Connelly, R.K. and Cokini, J.L., (2006b), "Mixing Simulation of a Viscous Newtonian Liquid in a Twin Sigma Blade Mixer", Advances in Polymer Technology, 25(3), 182-194.

Connelly, R.K. and Cokini, J.L., (2007), "Examination of the mixing ability of single and twin screw mixers using 2D finite element method simulation with particle tracking", J. Food Eng. 79, 956-969.

Crowe, C., M. Sommerfeld, and Y. Tsuji, (1998) Multiphase Flows with Droplets and Particles, CRC Press, Boca Raton, 1998.

Derksen, J., Van den Akker, H.E.A., (1999), "Large eddy simulations on the flow driven by a Rushton turbine", A.I.C.h.E.J., 45(2), 209-221.

Derksen, J.J., (2003), "Numerical simulation of solids suspension in a stirred tank", AIChE J., 49(11), 2700-2714

Derksen, J.J., (2001), "Assessment of large eddy simulations for agitated flows", Chem. Eng. Res. Des., 79(A9), 824-830

Derksen, J.J., Doelman, M.S., Van den Akker, H.E.A., (1999), “Three-dimensional LDA measurements in the impeller region of a turbulently stirred tank", Exp. Fluids, 27, pp. 522-532. 
Ding, J., and D. Gidaspow, A., (1990), "Bubbling Fluidization Model Using Kinetic Theory of Granular Flow", A.I.C.h.E.J., 36(4), 523-538.

Elgobashi, S. E., Abou-Arab, T. W., (1983), “A Two-Equation Turbulence Model for Two-Phase Flows", Phys. Fluids, 26, pp. 931-938.

Fluent, (2005), Fluent 6.2 User Guide.

Gavi, E., Marchisio D.L. and Barresi A.A., (2008), " On the importance of mixing for the production of nanoparticles ", J. .Disp. Sci. and Techn., 29 (4), 548-554

Giguere, R. Bertrand, F., and Tanguy, P.A. (2006), "A three-dimensional mesh refinement strategy for the simulation of fluid flow with a fictitious domain method", Comp. and Chem. Eng., 30 (3), 453-466.

Gimbun, J., Rielly, C.D. and Nagy, Z.K., (2009), "Modelling of Mass Transfer in GasLiquid Stirred Tanks Agitated by Rushton Turbine and CD-6 Impeller", $13^{\text {th }}$ Eur. Conf. Mixing, London

Guha, D., Ramachandran, P.A., Dudukovic, M.P. and Derksen, J.J., (2008), "Evaluation of large eddy simulation and Euler-Euler CFD models for solids flow dynamics in a stirred tank reactor", A.I.Ch.E.J., 54(3), 766-778.

Haque, J.N., Mahmud, T., and Roberts, K.J. (2006), " Modeling Turbulent Flows with Free-Surface in Unbaffled Agitated Vessels", Ind. Eng. Chem. Res, 45, 28812891.

Harris, C.K., Roekaerts, D., Rosendal, F.J.J., Buitendijk, F.G.J., Daskopoulos, P., Vreenegoor, A.J.N., Wang, H., (1996), "Computational fluid dynamics for chemical reactor engineering", Chem. Eng. Sci., 51, pp. 1569-1594.

Hartmann, H., Derksen, J.J., Montavon, C., Pearson, J., Hamill, I.S., van den Akker, H.E.A. (2004), "Assessment of large eddy and RANS stirred tank simulations by means of LDA", Chem. Eng. Sci., 59, pp. 2419-2432.

Hemrajani, R.R. and Tatterson, G.B. (2004) "Mechanically agitated vessels", (Chapter 6 in Handbook of industrial mixing, science and practice, ed., Paul, E. L., Atiemo-Obeng, V. A., Kresta, S. M.), John Wiley \& Sons, New Jersey, USA, pp. $345-390$.

Hirt, C.W, Nichols, B.D (1981), "Volume of fluid (VOF) method for the dynamics of free boundaries", J. Comp. Phys., 39, 201-225 
Huebner, K. H., Thornton, E. A., and Byron, T. D., The Finite Element Method for Engineers, $3^{\text {rd }}$ ed., Wiley Interscience(1995).

Iranshahi, A., Heniche, M., Bertrand, F. and Tanguy, P.A. (2006), "Numerical investigation of the mixing efficiency of the Ekato Paravisc impeller", Chem. Eng. Sci., 61(8), 2609-2617.

Issa, R.I. (1986) "Solution of Implicitly Discretized Fluid Flow Equations by Operator Splitting". J. Comp. Phys., 62, 40-65.

J. P. Vandoormaal and G. D. Raithby. (1984) "Enhancements of the SIMPLE Method for Predicting Incompressible Fluid Flows" Numer. Heat Transfer, 7,147-163.

Jahoda, M; Mostek, M; Kukukova, A, (2007), "CFD modelling of liquid homogenization in stirred tanks with one and two impellers using large eddy simulation", Chem. Eng. Res. Des., 85(A5), 616-625.

Jaworski, Z., Zakrzewska, B., (2002), "Modelling of the turbulent wall jet generated by a pitched blade turbine impeller: The effect of turbulence model", Chem. Eng. Res. Des., 80, pp. 846-854.

Jones, R.M., Harvey III, A.D., Acharya, S., (2001), "Two-equation turbulence modeling for impeller stirred tanks", J. Fluids Eng., 123, pp. 640-648.

Jongen, T. (2000), "Characterization of batch mixers using numerical flow simulations", A.I.C.h.E.J., 46(11), 2140-2150.

Khan, F.R., Rielly, C.D. and Brown, D.A.R., (2006), "Angle-resolved stereo-PIV measurements close to a down-pumping pitched-blade turbine", Chem. Eng. Sci., 61, 2799-2806

Khan, F.R., Rielly, C.D. and Hargrave, G.K., (2004) "A Multi-Block Approach to Obtain Angle-Resolved PIV Measurements of the Mean Flow and Turbulence Fields in a Stirred Vessel", Chem. Eng. Technol., 27, 264-269.

Kolmogorov, A.N., (1941), "The local structure of turbulence in incompressible viscous fluid for very large Reynolds numbers", Doklady Akademii Nauk SSR, 30, 301-305.

Kresta, S.M., \& Wood, P.E. (1991). "Prediction of the three-dimensional turbulent fow in stirred tanks. A.I.C.h.E.J., 37, 448-460. 
Laakkonen M., Moilanen P., Alopaeus V., Aittamaa J., (2007), "Modelling Local Gas-Liquid Mass Transfer in Agitated Vessels", Chem. Eng. Res. Des., 85(A5), 665-675.

Laakkonen M., Moilanen P., Miettinen T., Saari K., Honkanen M., Saarenrinne P., Aittamaa J., (2005), "Local bubble size distributions in agitated vessel comparison of three experimental techniques", Chem. Eng. Res. Des., 83, 5058.

Laakkonen, M., Moilanen, P., Alopaeus, V., Aittamaa, J., (2006), "Dynamic modeling of local reaction conditions in an agitated aerobic fermenter", AIChE Journal, 52, pp. 1673-1689.

Li M., White G., Wilkinson D., Roberts K. J. (2005), "Scale up study of retreat curve impeller stirred tank using LDA and CFD", Chem. Eng. J., 108, Issues 1-2, pp. 81-90.

Li, M., White, G., Wilkinson, D., Roberts, K.J., (2004), "LDA measurements and CFD modeling of a stirred vessel with a retreat curve impeller", Ind. Eng. Chem. Res., 43, pp. 6534-6547.

Li, Z.P., Gao, Z.M., Smith, J.M., (2007), "Large eddy simulation of flow fields in vessels stirred by dual Rushton impeller agitators", J.Chem.Eng.Jap., 40, 684691.

Leonard, B.P. (1979), "A stable and accurate convective modelling procedure based on quadratic upstream interpolation", Comput. Methods Appl. Mech. Eng., 19, 59-98.

Luo, J.Y. Issa, R.I. and A. D. Gosman, A.D. (1994) "Prediction of Impeller-Induced Flows in Mixing Vessels Using Multiple Frames of Reference", IChemE Symp. Ser. No. 136, pages 549-556.

Marchisio, D.L., Vigil, R.D. and Fox, R.O., (2003), "Quadrature method of moments for aggregation-breakage processes", J., Coll. and Interface Sci., 258(2), 322334.

Marshall, E.M., Bakker, A., (2004), "Computational Fluid Mixing”, (Chapter 5 in Handbook of industrial mixing, science and practice, ed., Paul, E. L., Atiemo- 
Obeng, V. A., Kresta, S. M.), John Wiley \& Sons, New Jersey, USA, pp. 257 344.

Montante, G and Magelli, F., (2005), "Modelling of solids distribution in stirred tanks: analysis of simulation strategies and comparison with experimental data", Int. J. of CFD, 19(3), 253-262

Montante, G., Horn, D. and Paglianti, A. (2008), "Gas-liquid flow and bubble size distribution in stirred tanks", Chem. Eng. Sci., 63(8), 2107-2118.

Montante, G., Paglianti, A. and Magelli F., (2004), "Liquid homogenization characteristics in vessels stirred with multiple rushton turbines mounted at different spacings. CFDstudy and comparison with experimental data", Chem. Eng. Res. Des., 82(A9), 1179-1187.

Montante, G., Paglianti, A. and Magelli F., (2007), "Experimental analysis and Computational modelling of Gas--liquid stirred vessels", Chem. Eng. Res. Des., 85(A5), 647-653

Nagata S. (1975), "Mixing: Principles and Applications", Halstead Press, Tokyo. Japan.

Navier, C.L.M.H, (1822), "Memoire sur les lois du mouvement des fluides", Mem. Acad. Sci. Inst. France, 6, 389-440.

Ng, K., Fentiman, N.J., Lee, K. C., Yianneskis, M., (1998), "Assessment of sliding mesh CFD predictions and LDA measurements of the flow in a tank stirred by a Rushton impeller", Chem. Eng. Res. Des. 76, pp. 737-747.

Nienow, A.W. (1997), "On impeller circulation and mixing effectiveness in the turbulent flow regime", Chem. Eng. Sci. 52, 2557-2565.

Norton, T. and Sun, D.W. (2006), "Computational fluid dynamics (CFD) - an effective and efficient design and analysis tool for the food industry: A review", Trends in Food Sci. \& Tech., 17, 600-620.

Ochieng, A. and Onyango, M.S. (2008), "Homogenization energy in a stirred tank", Chem. Eng. and Processing, 47(9-10), 1853-1860.

Patwardhan, A.W., (2001), "Prediction of flow characteristics and energy balance for a variety of downflow impellers", Ind. Eng. Chem. Res., 40, pp. 3806-3816. 
Ranade, V.V., Joshi, J.B., (1990), "Flow generated by a disc turbine. Part II.

Mathematical modelling and comparison with experimental data", Chem. Eng.

Res. Des., 68, pp. 34-50.

Revstedt, J., Fuchs, L., Tragardh, C., (1998), "Large eddy simulations of the turbulent flow in a stirred reactor", Chem. Eng. Sci., 53, pp. 4041-4053.

Rielly, C.D. \& Marquis, A.J. (2001) "A Particle's Eye View of Crystallizer Fluid Mechanics", Chemical Engineering Science, 56, pp 2475-2493

Rudman, M (1997), "Volume-tracking methods for interfacial flow calculations", Int. J. Num. Methods Fluids, 24, 671-691.

Ruszkowski, S. (1994) A rational method for measuring blending performance and comparison of different impeller types. In Proc. 8th Europ. Mixing Conf., pp. 283-291. Inst. Chem. Engrs. Rugby, U.K.

S. V. Patankar. Numerical Heat Transfer and Fluid Flow. Hemisphere, Washington D.C., 1980.

Sbrizzai, F., Lavezzo, V., Verzicco, R., Campolo, M., Soldati, A., (2006), "Direct numerical simulation of turbulent particle dispersion in an unbaffled stirred-tank reactor", Chem. Eng. Sci., 61, pp. 2843-2851.

Schäfer M, Yianneskis M, Wächter P, Durst F (1998), "Trailing vortices around $45^{\circ}$ pitched-blade impeller", AIChE J, 44, pp. 1233-1245.

Shih, T.H. Liou, W.W., Shabbir, A., Yang, Z. and Zhu, J., (1995), "A new k-E eddy viscosity model for high Reynolds number turbulent flows", Comput. Fluids 24(3), 227-238

Simonin, O., Viollet, P.L., (1990), "Prediction of an oxygen droplet pulverization in a compressible subsonic coflowing hydrogen flow", ASME, Fluids Engineering Division (Publication), FED 91, pp. 73-82.

Spalart, P.R., Jou, W.-H., Strelets, M., Allmaras, S.R., (1997), "Comments on the Feasibility of LES for Wings, and on a Hybrid RANS/LES Approach", Advances in DNS/LES, $1^{\text {st }}$ AFOSR Int. Conference on DNS/LES, 4-8 Aug. Greyden Press, Columbus, $\mathrm{OH}$.

Stokes, G.G., (1845) "On the theories of internal friction of fluids in motion", Trans. Camb. Phil.. Soc. 8, 287-305. 
Sun, D.W, (2007), "Computational Fluid Dynamics in Food Processing", CRC Press, Taylor \& Francis Group, Boca Raton, FI, USA.

Tyagi, M., Roy, S. Harvey, A.D., (2007), "Simulation of laminar and turbulent impeller stirred tanks using immersed boundary method and large eddy simulation technique in multi-block curvilinear geometries", Chem. Eng. Sci., 62 (5), 13511363.

Vandoormaal J.P. and Raithby G.D., (1984), "Enhancements of the SIMPLE method for predicting incompressible fluid flows", Numer. Heat Transfer, 7, 147-163.

Van't Riet, K. and Smith, J. M., (1975), "The trailing vortex system produced by Rushton turbine agitators", Chem. Eng. Sci., 30, 1093-1105.

Venneker, B.C.H., Derksen, J.J., Van den Akker, H.E.A., (2002), "Population balance modeling of aerated stirred vessels based on CFD", A.I.C.h.E.J.., 48, pp.673685.

Versteeg, H.K. and Malalasekara, W., (2007), "An introduction to computational fluid dynamics. The finite volume method", Pearson Education, Harlow, UK.

Verzicco, R., Fatica, M., laccarino, G., Orlandi, P., (2004), "Flow in an impellerstirred tank using an immersed-boundary method", A.I.C.h.E.J., 50, pp. 11091118.

Wei, H., Zhou, W. and Garside, J. (2001), " Computational Fluid Dynamics Modeling of the Precipitation Process in a Semibatch Crystallizer", Ind. Eng. Chem. Res., 40, 5255-5261

Wu, H. and Patterson, G. K., (1989), Laser Doppler measurements of turbulent flow parameters in a stirred mixer, Chem. Eng. Sci., 44, 2207-2221

Yakhot, V. and S. A. Orszag, (1986), "Renormalization Group Analysis of Turbulence: I. Basic Theory", J. Sci. Computing, 1(1), 1-51.

Yapici, K. ,Karasözen, K., Schäfer, M. and Uludag, Y., (2008), "Numerical Investigation of the Effect of the Rushton Type Turbine Design Factors on Agitated Tank Flow Characteristics", Chem. Eng. \& Processing, 47, 1346-1355, 2008 
Yeoh, S.L., Papadakis, G. Yianneskis, M. (2005) "Determination of mixing time and degree of homogeneity in stirred vessels with large eddy simulation", Chem. Eng. Sci. 60, $2293-2302$

Yeoh, S.L., Papadakis, G., Lee, K.C., Yianneskis, M., (2004), "Large Eddy Simulation of turbulent flow in a Rushton impeller stirred reactor with slidingdeforming mesh methodology", Chem. Eng. Technol., 27, pp. 257-263.

Yianneskis, M., Popiolek, Z., Whitelaw, J.H., (1987), "Experimental study of the steady and unsteady flow characteristics of stirred reactors", J. Fluid Mech., 175, pp. 537-555.

Yianneskis, M., Whitelaw, J.H., (1993), "On the structure of the trailing vortices around Rushton turbine blades", Chem. Eng. Res. Des., 71, pp. 543-550.

Yimer, I., Campbell, I. and Jiang, L-Y., (2002), "Estimation of the turbulent Schmidt number from experimental profiles of axial velocity and concentration for highReynolds-number jet flows", Can Aeronaut Space J, 48, 195-200.

Zwietering T.N. (1958), "Suspending of solid particles in liquid by agitators", Chem. Eng. Sci., 8, pp.244-253 


\section{COMPUTATIONAL FLUID MIXING}

Figures and captions

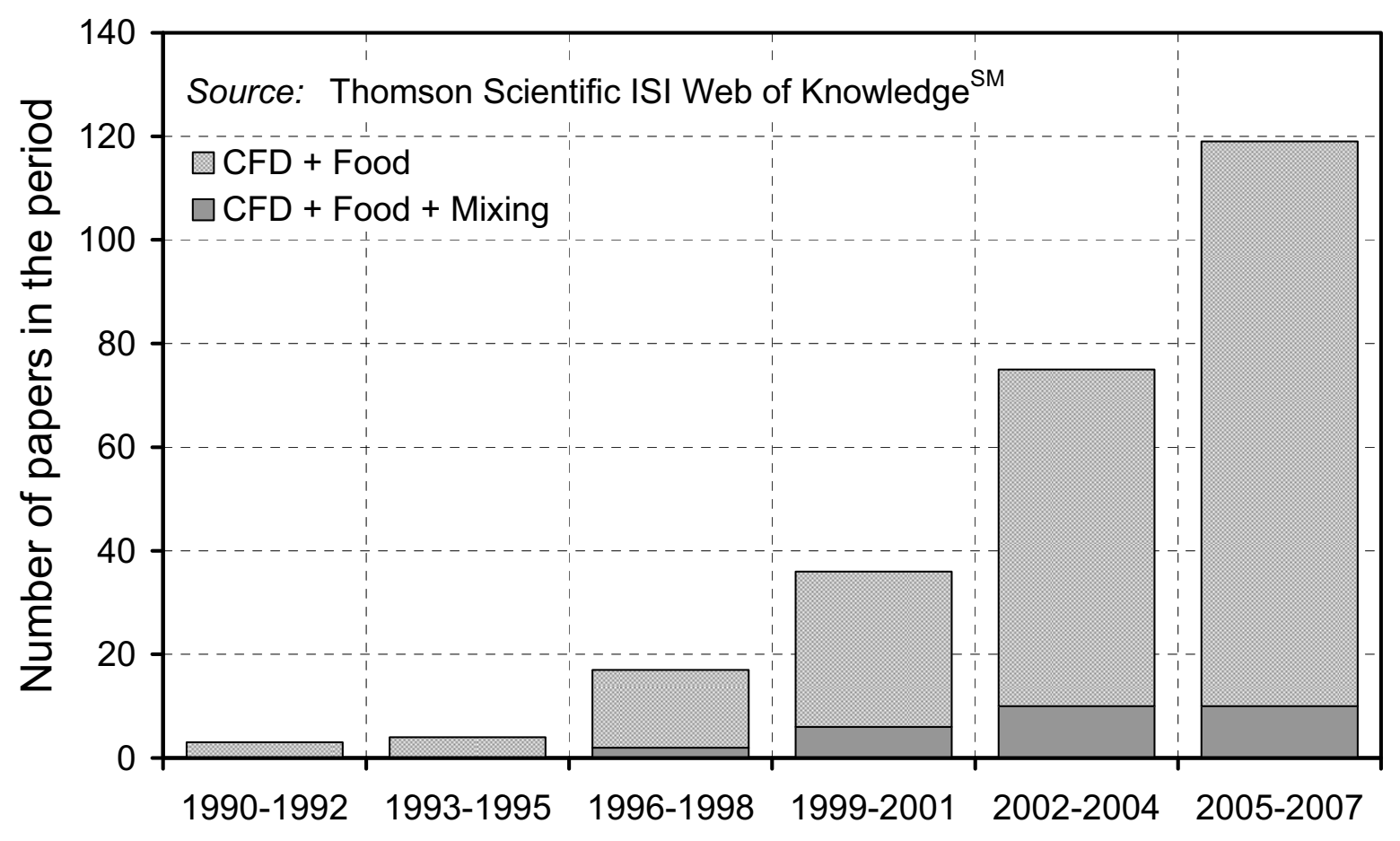

Figure 8.1. Number of papers published on food and CFD from 1990 to 2007. Update and enhanced version first shown by Norton \& Sun (2006). 


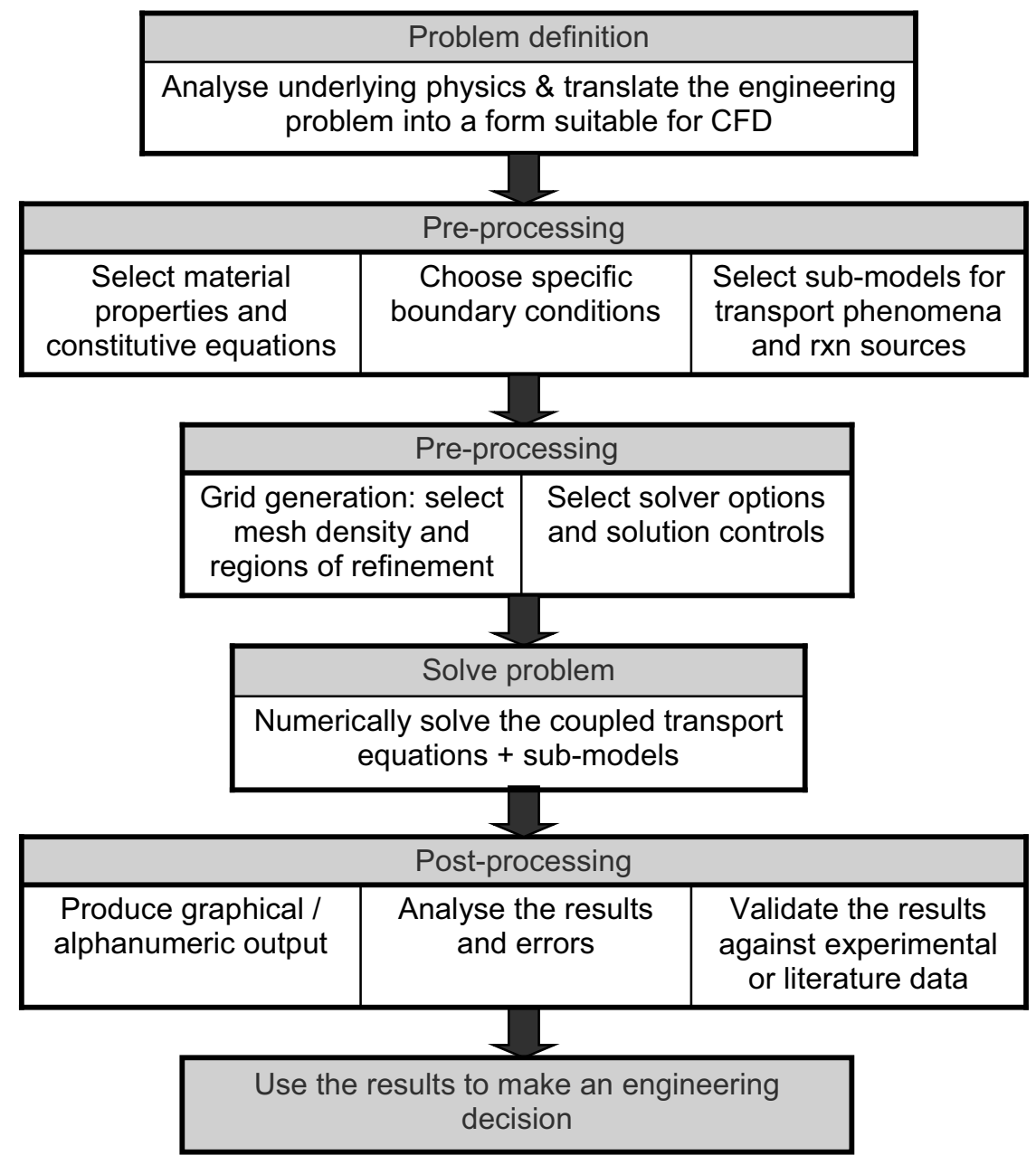

Figure 8.2. Procedure for the solution of a mixing problem via CFD simulation. 


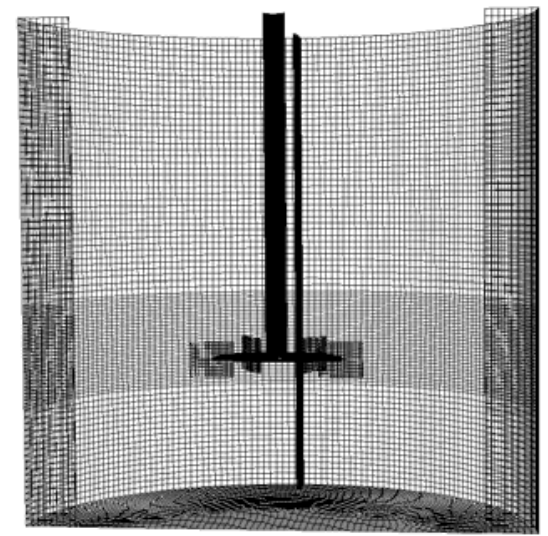

Figure 8.3. Computational mesh shown on the wall of a stirred vessel containing a Rushton disk turbine (Gimbun, 2008) 


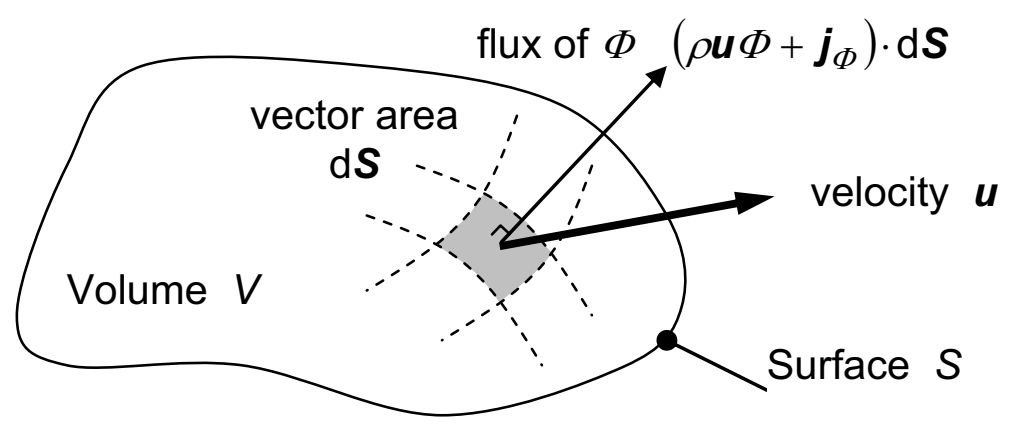

Figure 8.4. Fixed control volume $V$ bounded by surface $S$ used for conservation balances. 


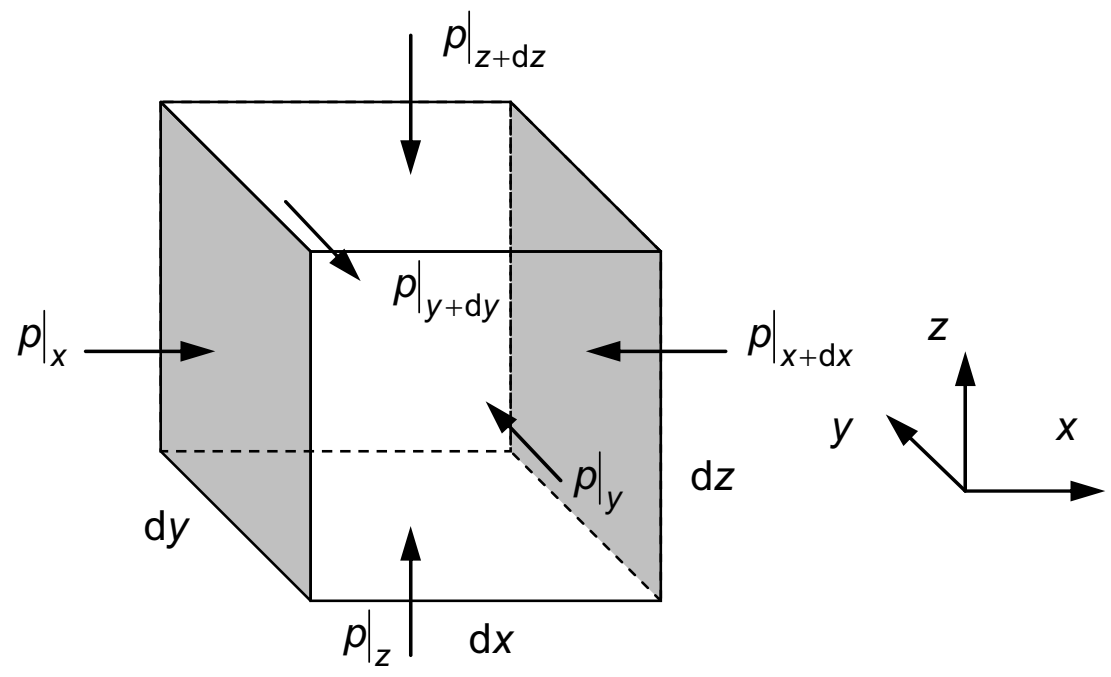

Figure 8.5 Cartesian fluid element with a pressure gradient giving rise to normal force differences acting on its faces. 


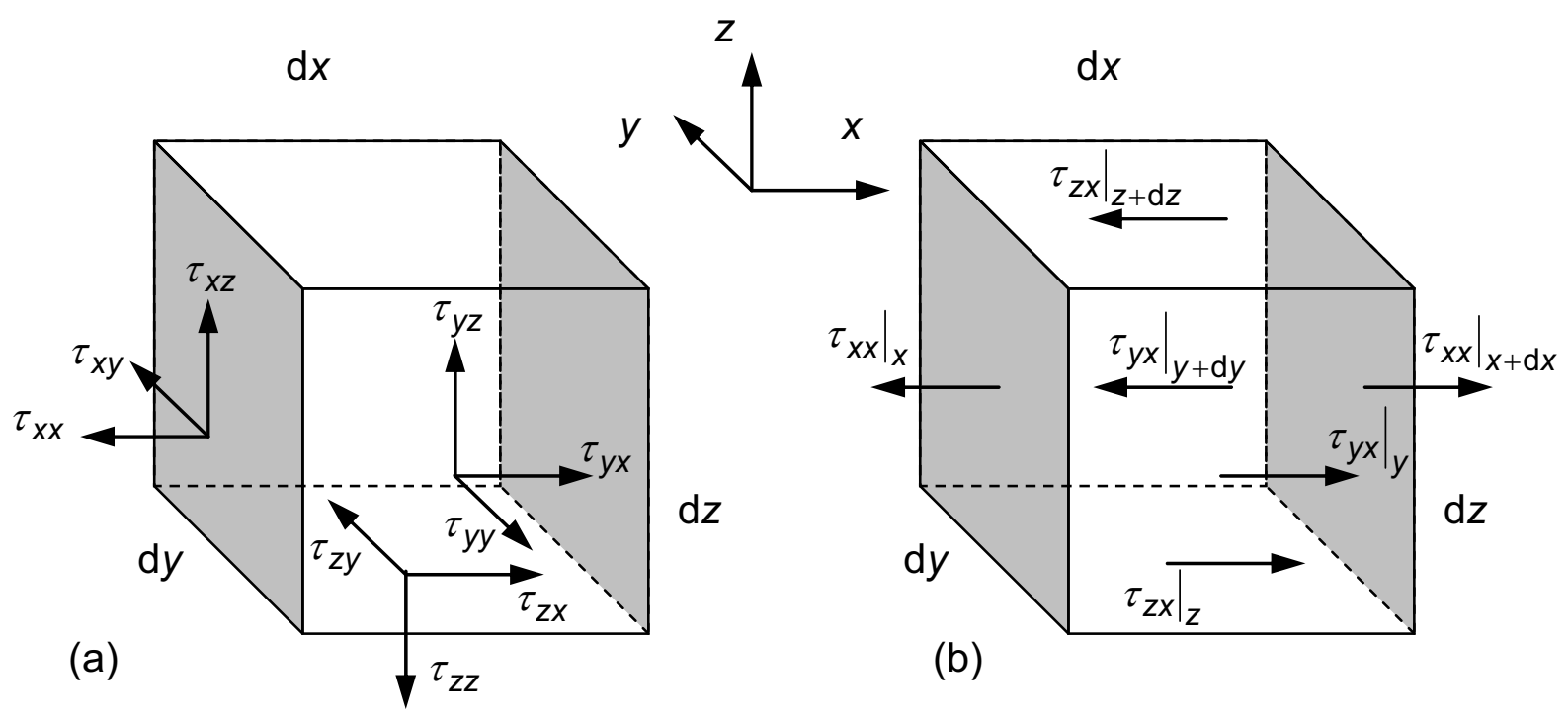

Figure 8.6.Cartesian fluid element showing (a) the stresses acting on three of its faces and (b) the stresses that contribute to the $x$-direction force 


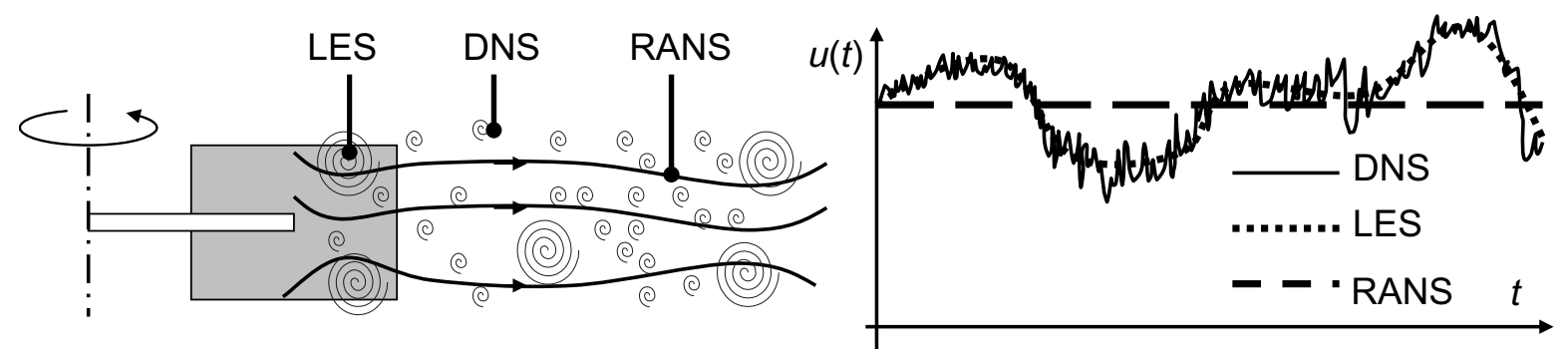

Figure 8.7. Schematic representation of the various levels of turbulence model for implementation in CFD calculations. 


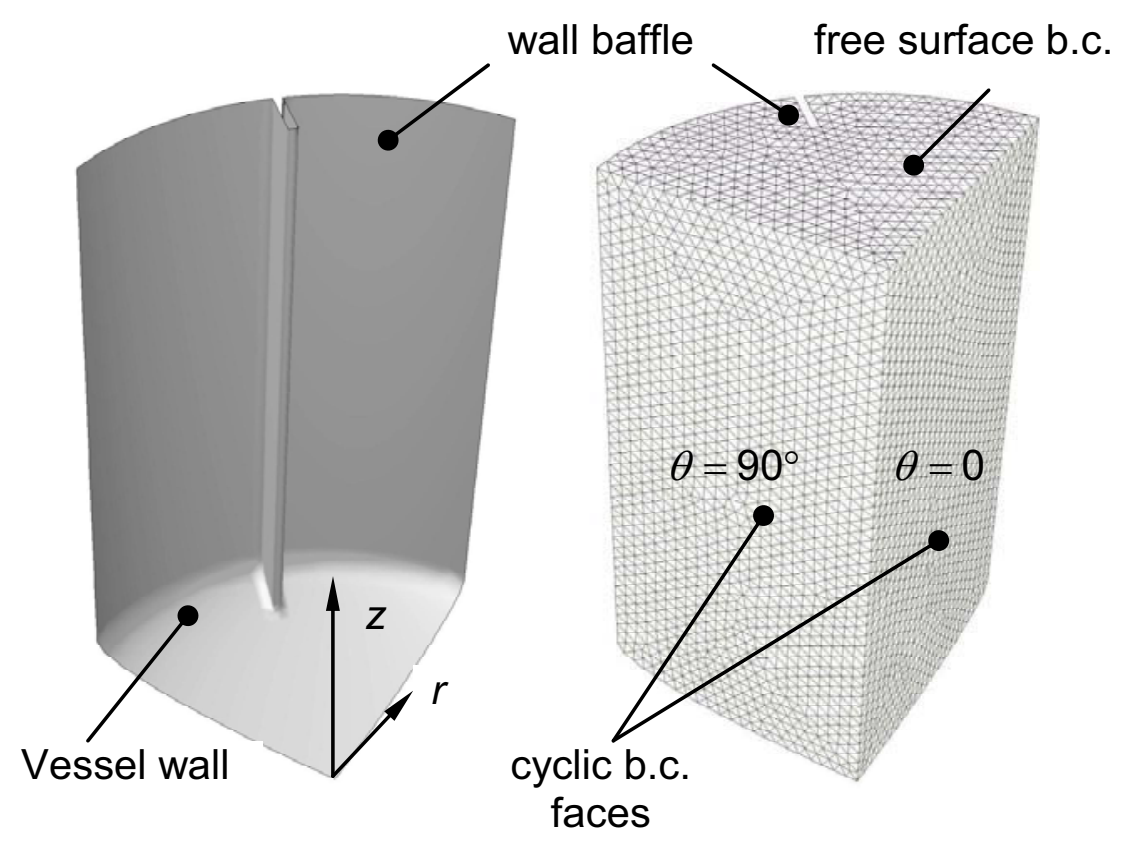

Figure 8.8. Use of cyclic boundary conditions to reduce computational costs of simulating steady-state, fully baffled flows 


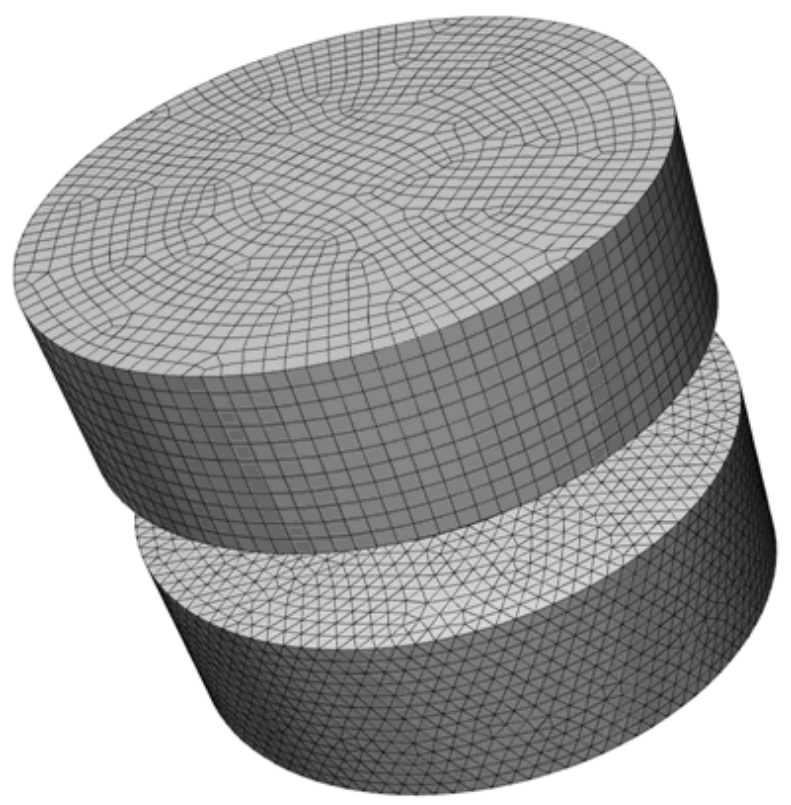

Figure 8.9. An illustration of the difference between a hexahedral unstructured mesh (upper) and a tetrahederal unstructured mesh (lower). 

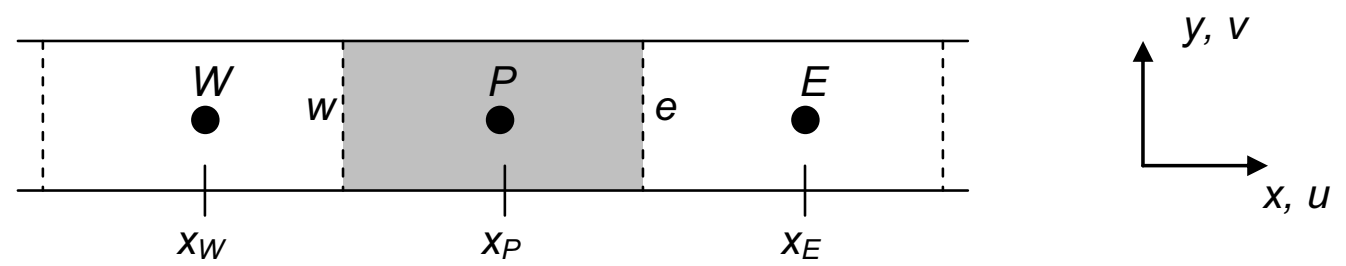

Figure 8.10. A simple one-dimensional grid, for finite-volume analysis. 


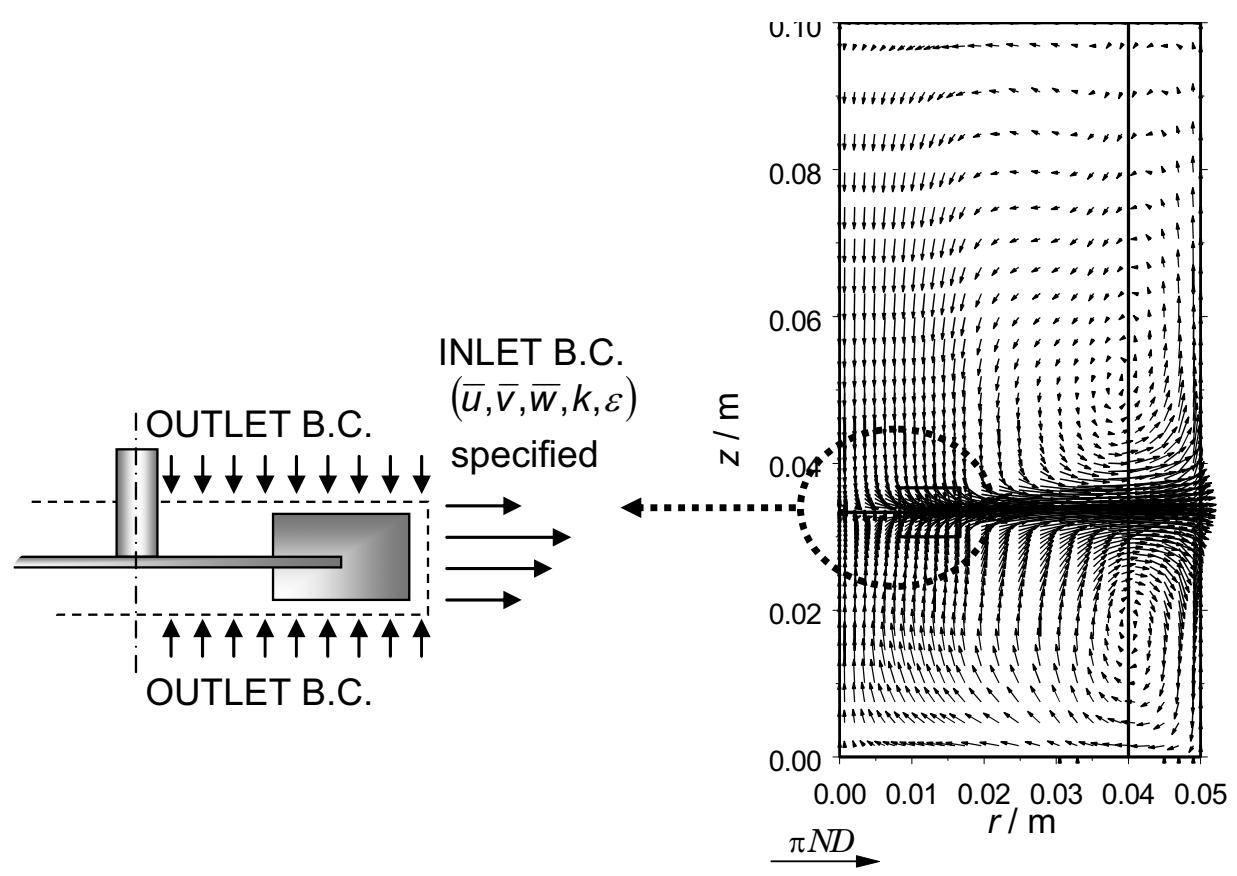

Figure 8.11. Black-box approach to impeller modelling using prescribed boundary conditions on the swept surface of the blades. 


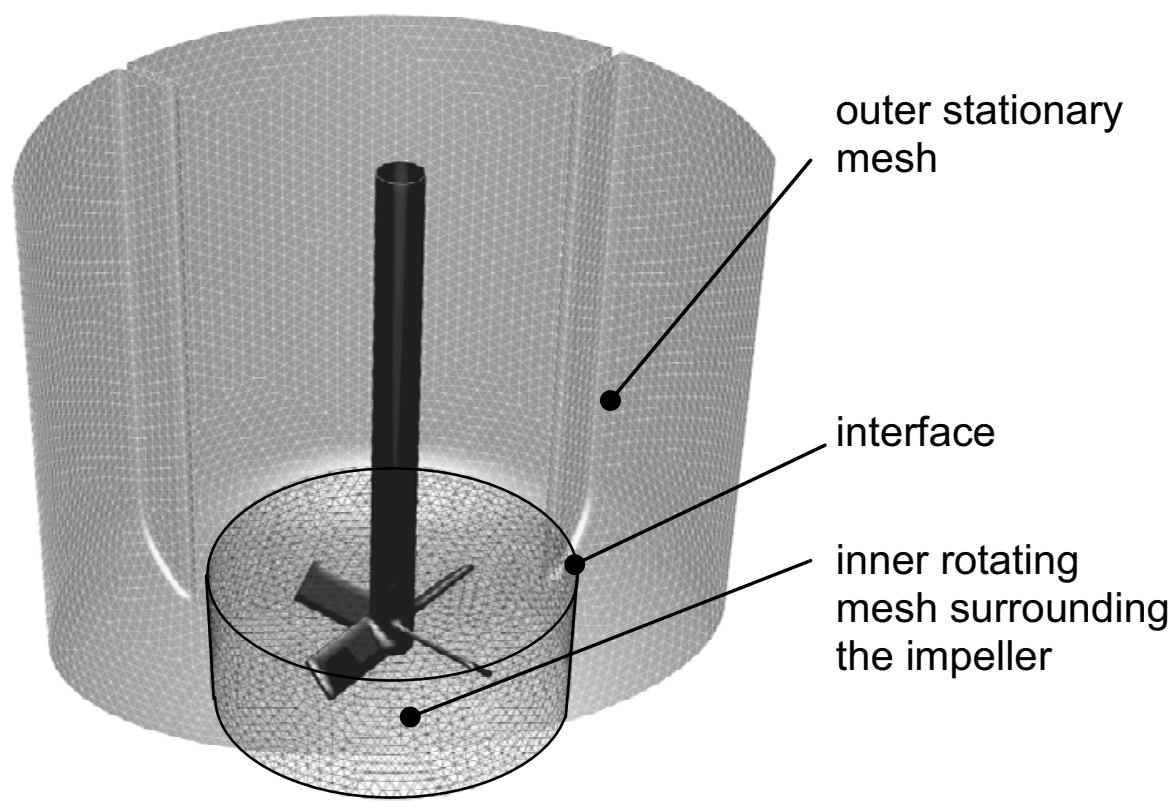

Figure 8.12. Multiple reference frames representation using a rotating grid around the impeller itself and a stationary grid to mesh the walls and baffles. 


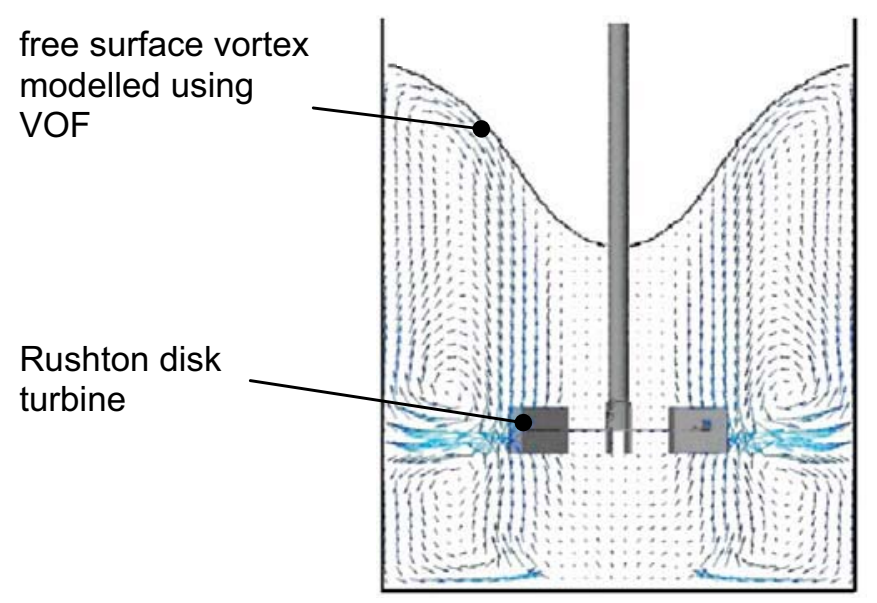

Figure 8.13. Mean velocity vector map and surface profile for an unbaffled tank, predicted by Haque et al. (2006) using a VOF model. [Reprinted with permission from Haque et al. (2006). Copyright American Chemical Society] 


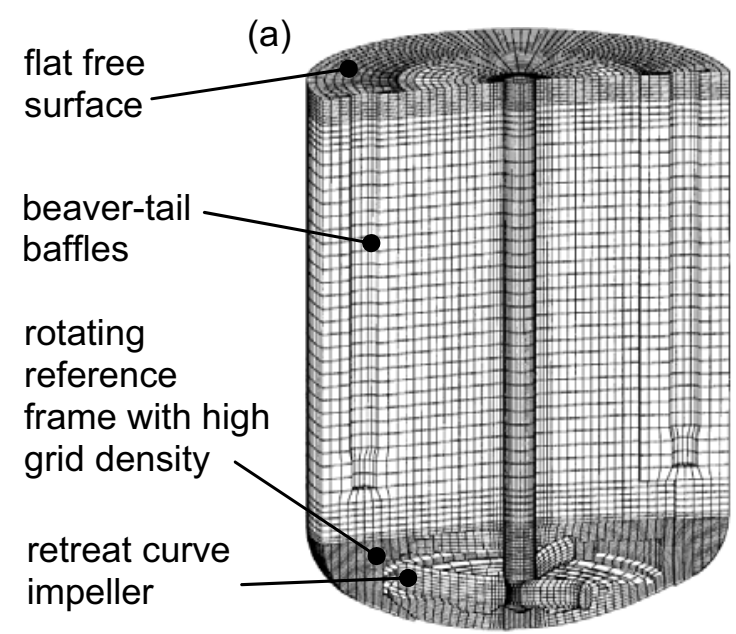

(b)

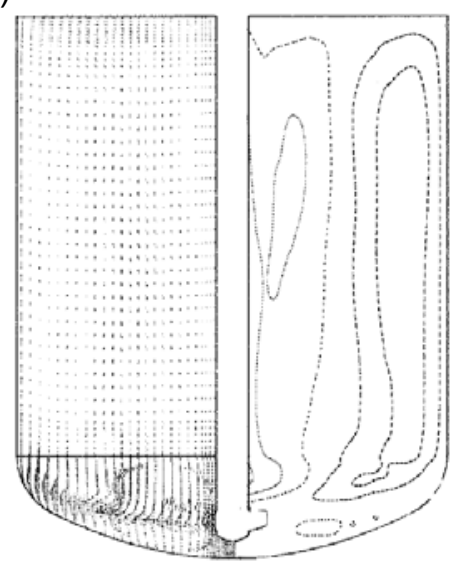

Figure 8.14. (a) grid and geometry used by Campolo et al (2002) and Campolo and Soldati (2002) to model an industrial scale vessel (b) the mean velocity field and streamlines for the retreat curve impeller. [Reprinted with permission from Campolo et al (2002) and Campolo and Soldati (2002). Copyright American Chemical Society] 


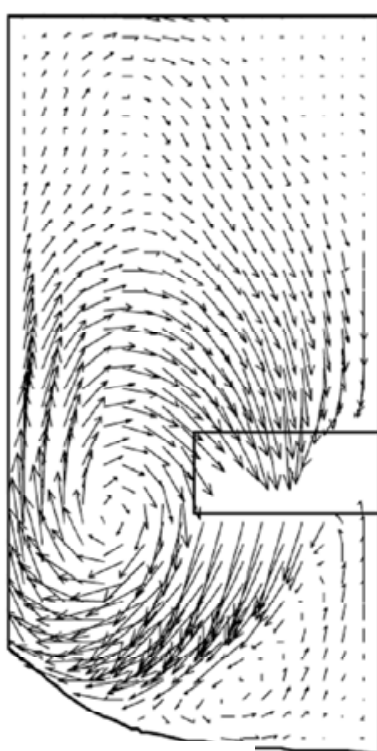

(a)

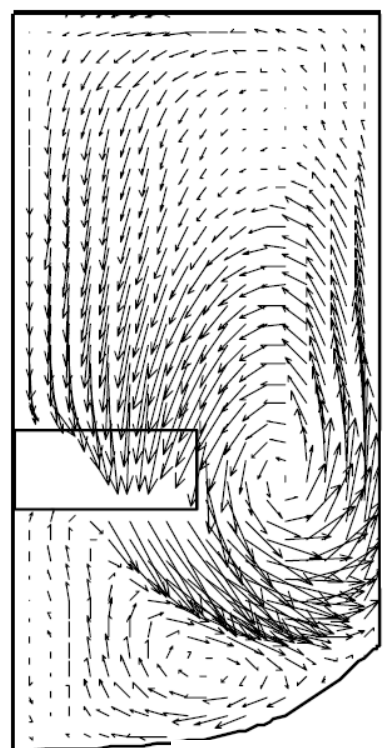

(b)

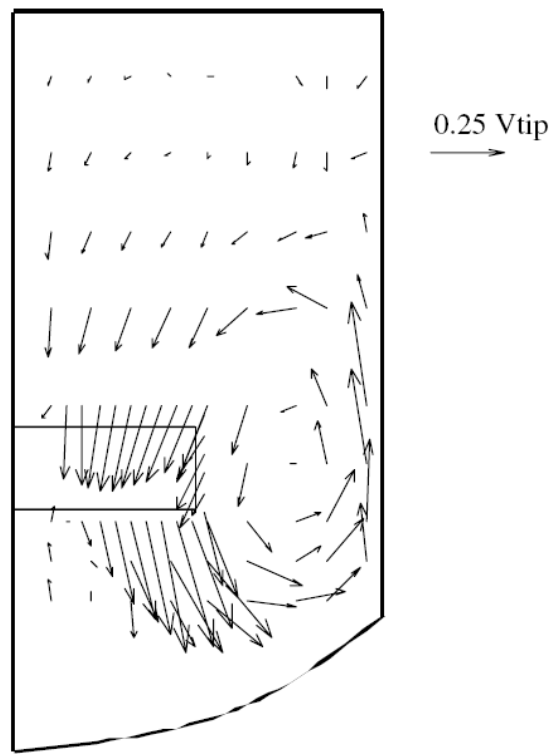

(c)

Figure 8.15.Standard $k-\varepsilon$ and higher-order upwind discretisation (a) MRF model and (b) SM model, compared to LDA data (Aubin et al, 2004) [Reprinted from Aubin et al (2004) with permission from Elsevier.] 

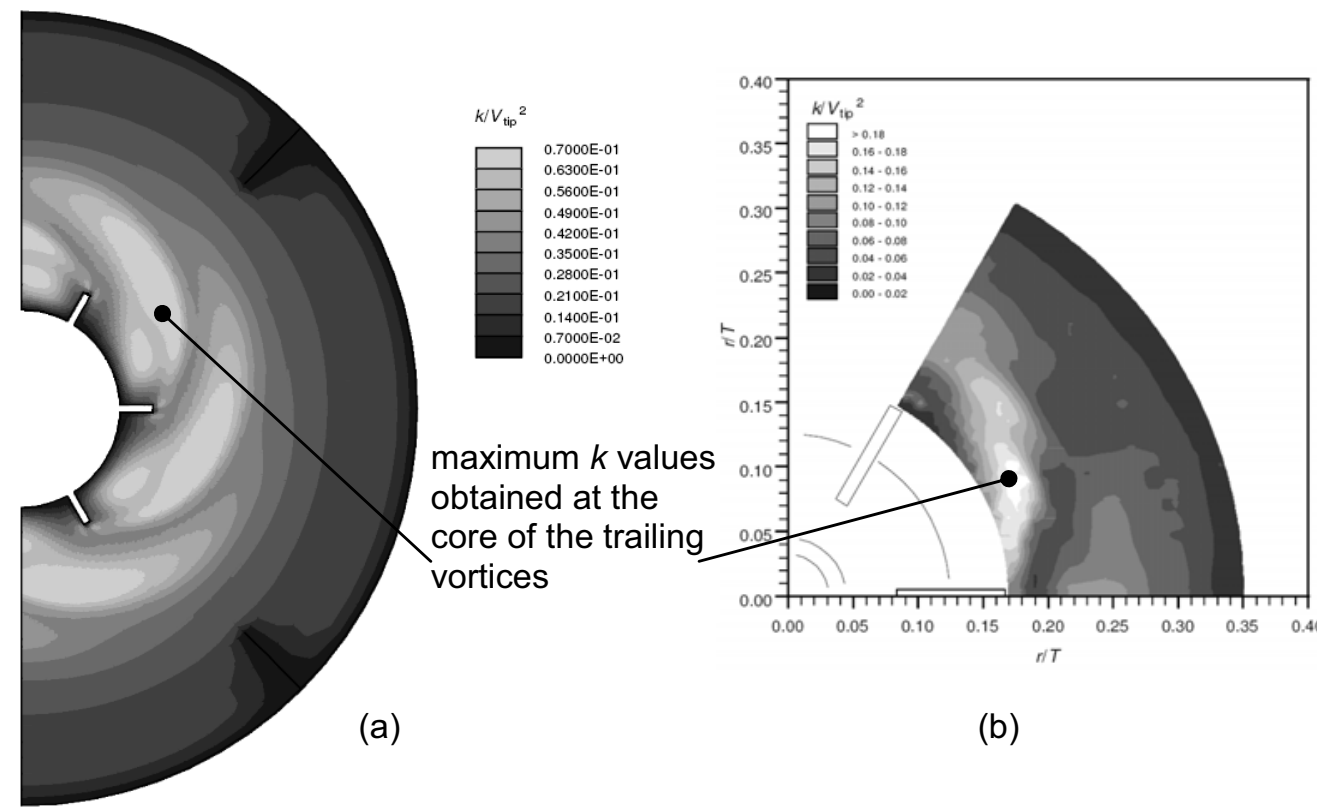

(b)

Figure 8.16. (a) CFD SM simulations of a Rushton disk turbine performed by Ng et al (1998) using a standard $k-\varepsilon$ RANS model (b) comparison with angle-resolved LDA measurements [Reprinted from $\mathrm{Ng}$ et al (1998) with permission from Elsevier.] 
a
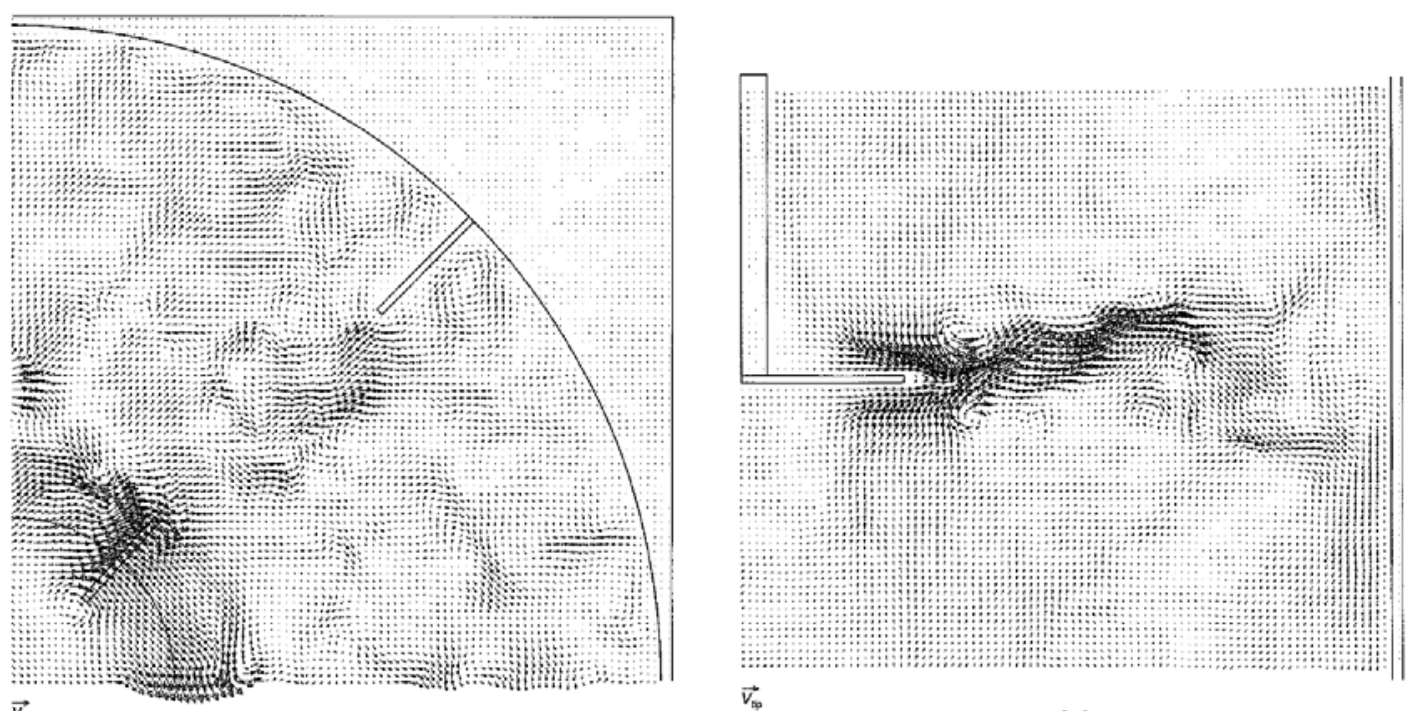

Figure 8.17. (a) LES simulations of a Rushton disk turbine performed by Derksen and van den Akker (1999) [Reproduced with permission from John Wiley \& Sons, Inc.] 

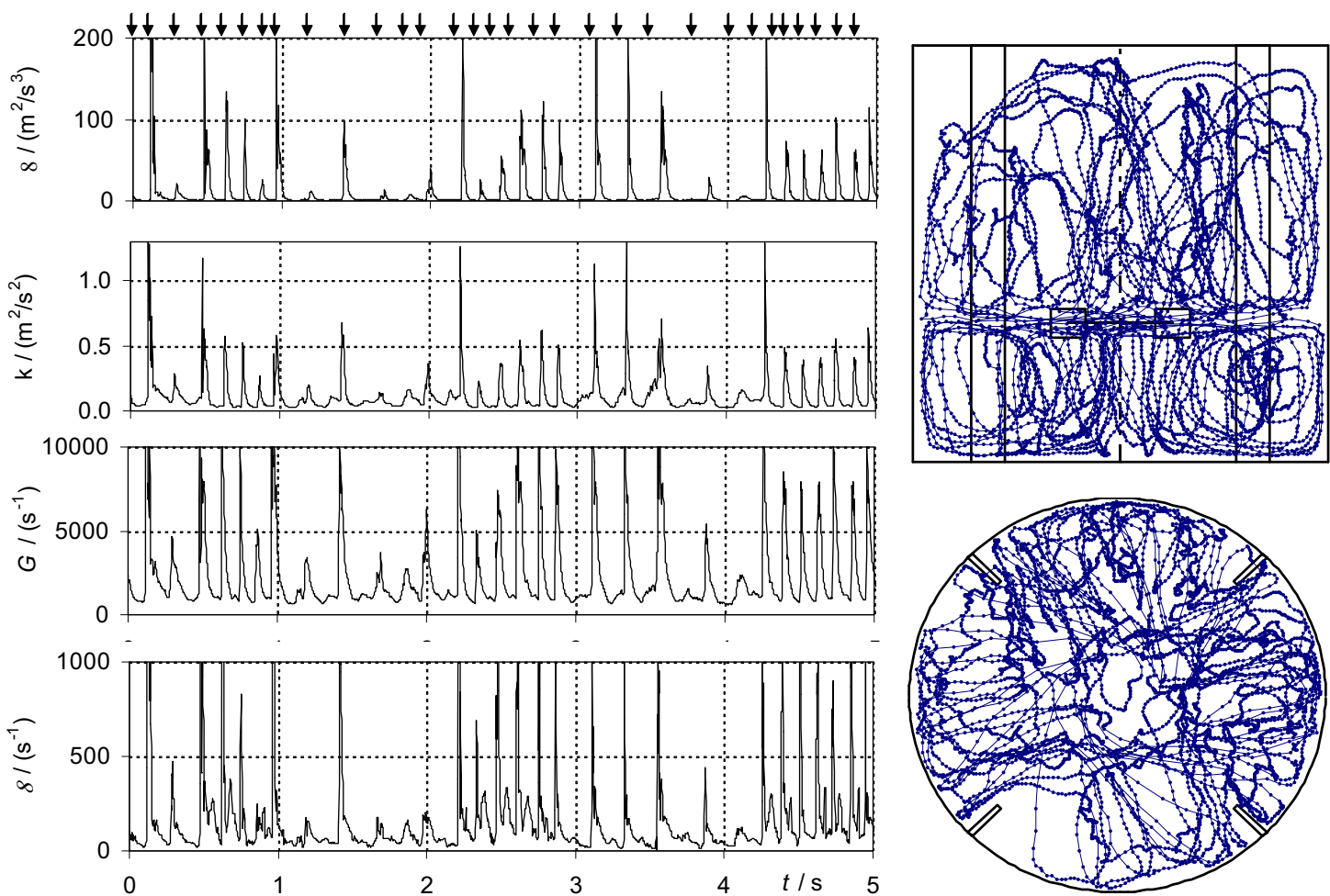

Figure 8.18. Lagrangian particle tracking in a stirred vessel showing particle tracks and time histories of dissipation rate, $\varepsilon$, turbulence kinetic energy, $k$, Kolmogorov strain rate, $G$, and mean flow strain rate, $\gamma$, arrows indicate times when the particle is in the impeller plane 

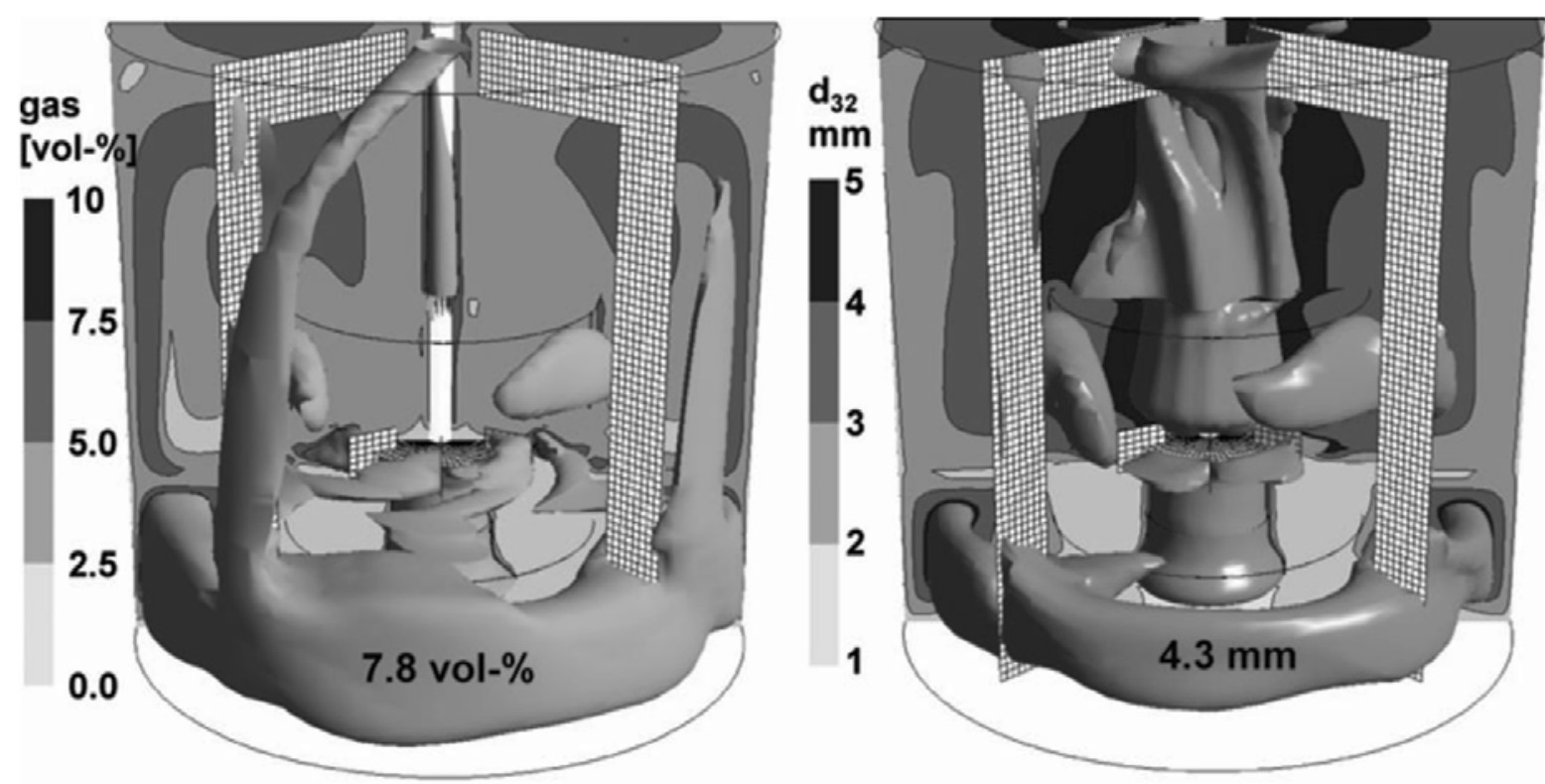

Figure 8.19. Local gas holdup (vol\%) and Sauter mean bubble diameter $d_{32}(\mathrm{~mm})$ in an agitated gas-liquid flow (Laakonen et al., 2007) [Reprinted from (Laakonen et al., 2007) with permission from Elsevier.] 

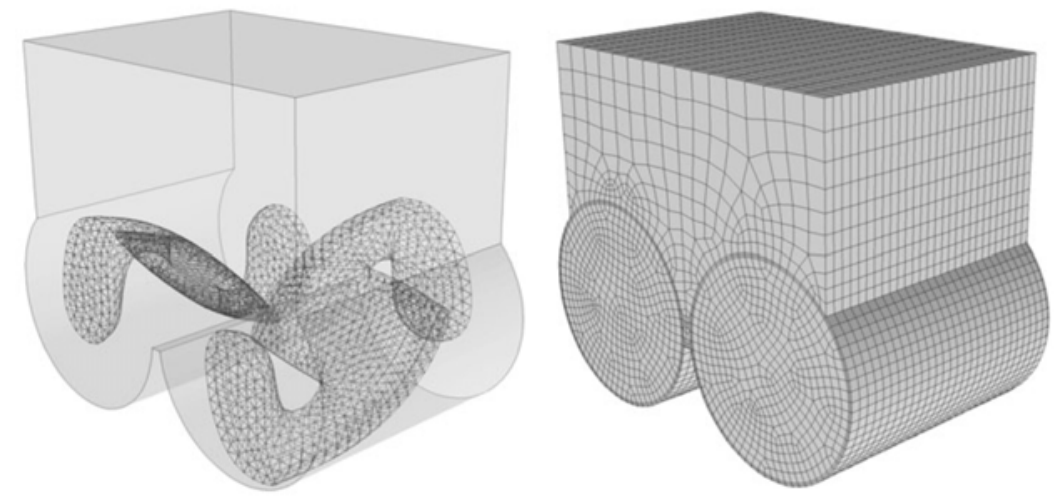

Figure 8.20.Mesh used in the POLYFLOW simulation of a Farinograph sigma-blade dough mixer (Connelly and Kokini,, 2006a) [Reproduced with permission from John Wiley \& Sns, Inc.] 


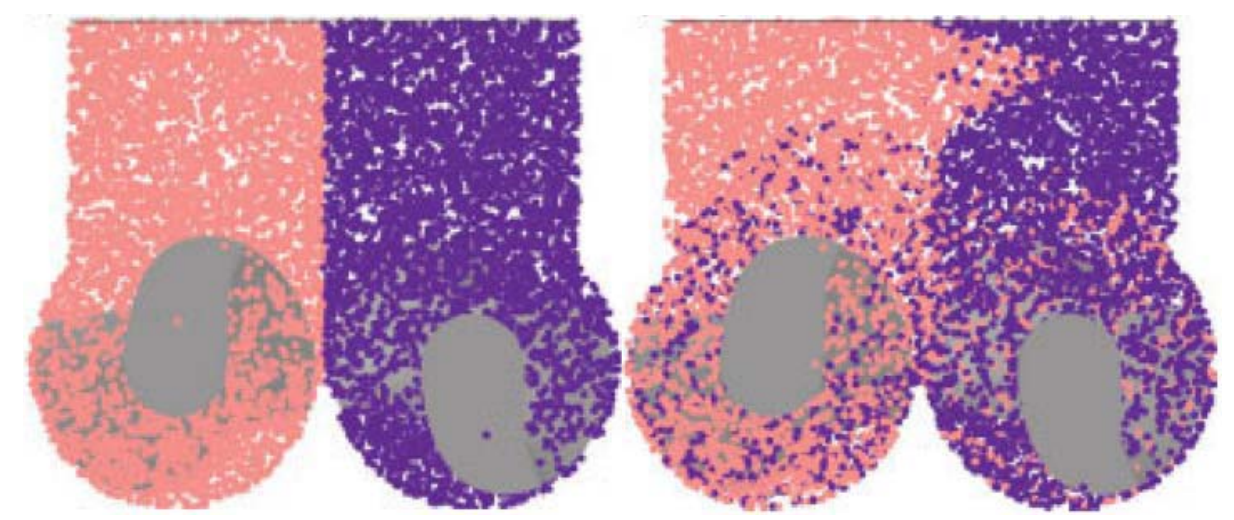

Figure 8.21.Assessment of mixing from particle tracking in the POLYFLOW simulation of a Farinograph sigma-blade dough mixer (Connelly and Kokini,, 2006b): (a) the initial configuration of 10000 particles and (b) their positions after 3 revolutions of the impeller [Reproduced with permission from John Wiley \& Sns, Inc.] 